\title{
The Indo-Europeanization of the World from a Central Asian Homeland: New Approaches, Paradigms and Insights from Our Research Publications on Ancient India
}

\author{
Sujay Rao Mandavilli (Corresponding author) \\ Independent Scholar, India \\ E-mail: sujayrao2012@gmail.com
}

Received: August 3, 2015 Accepted: September 8, 2015 Published: September 10, 2015

doi:10.5296/jsss.v3i1.8278 URL: http://dx.doi.org/10.5296/jsss.v3i1.8278

\begin{abstract}
In this paper, we bring together the concepts put forth in our previous papers and throw new light on how the Indo-Europeanization of the world may have happened from the conventional Central Asian homeland and explain the same using maps and diagrams. We also propose the 'Ten modes of linguistic transformations associated with Human migrations.' With this, the significance of the proposed term 'Base Indo-European' in lieu of the old term 'Proto Indo-European' will become abundantly clear to most readers. The approaches presented in this paper are somewhat superior to existing approaches, and as such are expected to replace them in the longer run. Detailed maps and notes demonstrating and explaining how linguistic transformations might have taken place in South Asia are available in this paper as understood from our previous research papers, and scholars from other parts of the world are invited to develop similar paradigms with regard to their home countries as far as the available data or evidence will allow them. This will help piece together a gigantic jig-saw puzzle, and lead to a revolution of sorts in the field, leading to a ripple-effect that will strongly impact several other related fields of study as well. We also re-emphasize our epigrammatic catch-phrases 'The Globalization of Science' and 'Scientific Progress at the Speed of Light', and attempt to show how the former will inexorably lead to the latter. This is done in a respectable level of detail, as zany and theoretical concepts gain respectability only if corroborated with real-world data from across the world. The end-result will be a transformation and a revolution in human knowledge, with inevitable cascading changes in cultural and social paradigms and relationships across nationalities and cultures, and rich rewards for scholars and students of Indo-European studies across the world.
\end{abstract}


Keywords: Aryan, Dravidian, Harappan, Human Migrations, India, Indo-European, PIE, Sanskrit

\section{The Indo-European Question and Its History}

The Indo-European question has been the mother of all historical puzzles ever since Sir William Jones, then a British judge in India, well-acquainted with Indian languages and literature, and also one of co-founders of the Asiatic Society of Bengal, delivered a lecture in Calcutta on the third anniversary celebrations of the founding of the afore-said society held on the second of February, 1786, in which he put forth his theory that the Indo-Aryan languages spoken in India were related to those in Europe and had a common ancestor, known as Proto Indo-European. In this very speech, he stated: "The Sanskrit language, whatever be its antiquity, is of a wonderful structure; more perfect than the Greek, more copious than the Latin, and more exquisitely refined than either, yet bearing to both of them a stronger affinity, both in the roots of verbs and in the forms of grammar, than could possibly have been produced by accident; so strong indeed, that no philologer could examine them all three, without believing them to have sprung from some common source, which, perhaps, no longer exists: there is a similar reason, though not quite so forcible, for supposing that both the Gothic and the Celtic, though blended with a very different idiom, had the same origin with the Sanskrit; and the old Persian might be added to the same family, if this were the place for discussing any question concerning the antiquities of Persia." Although some early missionaries such as Filippo Sassetti had noted the similarities between European and Indian languages much earlier, as also had James Burnett aka Lord Monboddo in the Eighteenth century before Jones, this speech effectively laid the foundation for Indo-European studies as it was widely quoted in other publications, and had a major impact at that time.

Another key reason for the widespread interest in the Indo-European problem was the fact that Indo-European languages were widely spoken in Europe, North America, South America, Australia and other influential regions of the world. Yet another reason for this interest was that this language group was associated with Ancient Greece, Ancient India and Ancient Iran too. But perhaps the most important reason for this interest was the sheer complexity of this problem, the vast distances involved, the spatial spread of Indo-European languages, and the sheer magnitude of the impact it came to have on various cultures across the world: it is likely that this purported language family was spread by small groups of non-literate, horse-riding nomads radiating in different directions from the Indo-European homeland. Jones' ideas found support in other scholars, and the foundation was eventually laid for the study of comparative philology or historical linguistics. In 1816, the German scholar Franz Bopp published his observations on the grammar of Indo-European. This work was further extended by August Scheleicher in 1852. Jacob Grimm also demonstrated the regularity of sound shifts of Indo-European languages as they distanced from each other. The latter came to be known as Grimm's Law of transformational grammar.

\subsection{The Linguistic Sub-Groups Forming a Part of the Indo-European Family}

The spread of IE was so complex, it spawned ten major linguistic sub-groups which are as below. These classifications have been proposed by scholars such as August Schleicher, E. 
Sturtevant, Gamkrelidze, Ivanov, Vladimir Georgiev and Eric Hamp.

(a) Indo-Aryan languages of North India and Sri Lanka (Also commonly known as Indic) is one sub-group. Of this, Vedic Sanskrit is the most archaic, with hymns believed to have been orally transmitted from as early as 1650 BC -1380 BC. Modern Indo-Aryan languages include Hindi, Bengali, Assamese, Nepali, Marathi, Punjabi and Gujarati.

(b) Iranian languages of Iran constitute another sub-group. Modern living descendants of this language group include languages such as Farsi (from Old Persian), Pashto and Kurdish. Proto-Iranian, if at all such a language existed, is dated at $1500 \mathrm{BC}$, but the early history or Iran is notoriously unreliable, much worse than India's even. Some other scholars think the oldest portion of the Gatha of the Avesta dates to around 1000 BC. However, the dates arrived at for the entry of an ancestor of Brahmi into India and its split up from early West Asian alphabetic scripts in our papers using independent methods, seem to vindicate our model on cultural contacts between Ancient India and Iran down to the very last century, and this puts 'Aryan' presence in Iran before 1700 BC.

(c) Romance languages such as Spanish, French, Italian (These are believed to have been influenced heavily by Latin) are yet another sub-group of Indo-European. Oscan and Umbrian were other extinct languages of this group.

(d) Greek or Hellenic languages (From Ancient Greek) which is attested from Linear B inscriptions dating back to $1400 \mathrm{BC}$ and later Homeric texts, and has essentially been one language with many dialects throughout its long history.

(e) Germanic languages such as English, Dutch, German (from Gothic which is an extinct East Germanic language and Old English, which is a West Germanic language. The nature of relationship between these and modern languages is to be probed further). The earliest evidence of Germanic languages comes from the Runic inscriptions of the second century.

(f) Celtic languages such as Irish and Welsh (These are believed to have been derived from Ancient Celtic. The relationship between this sub-group and IE may need to be probed further) form a branch of the Indo-European family. These are chiefly found in the present-day United Kingdom. However, traces of these are found in Gaulish and Celtiberian inscriptions dating to the $3^{\text {rd }}$ Century BC. Old Irish is another important language of this family. Welsh is attested from around the $8^{\text {th }}$ century.

(g) Baltic languages such as Lithuanian, Latvian, and the extinct Old Prussian which are attested from $1400 \mathrm{AD}$.

(h) Slavic languages including the early medieval language Old Church Slavonic and the modern Slavic languages (Russian, Polish, Czech, etc.). The oldest form of this language is attested from $900 \mathrm{AD}$.

(i) Armenian in both Classical and modern forms constitutes another branch of the Indo-European family. This branch demonstrates some diversity in dialects.

(j) Albanian in two main dialect groups i.e. Geg and Tosk dating to $1500 \mathrm{AD}$ is a branch of the Indo-European family, and quite possibly the youngest. Some scholars believe it has some connection with languages such as Illyrian or Thracian but such theories are controversial. 
(k) Anatolian, including Hittite, Palaic, Luwian, Lydian, Lycian, Milyan, Carian and other languages of ancient Asia Minor (this is a mostly extinct group) form another sub-group. Hittite and Palaic are the oldest attested languages of the Indo-European language family, with records dating back to $1800 \mathrm{BC}$. All these languages were discovered in the early twentieth century. Hittite was also the administrative language of Hatti, an ancient kingdom and was recorded in Cuneiform tablets. All these languages were extinct by late Hellenistic times.

(1) Tocharian, consisting of two closely inter-related languages (Tocharian A and B) with documents dating from the $6^{\text {th }}$ to the $8^{\text {th }}$ Century AD, known from archaeological sites on the Silk Road, in the Chinese province of Xinjiang and Chinese Turkestan (This group became extinct by the $10^{\text {th }}$ Century AD and these documents were discovered only in the $19^{\text {th }}$ and the $20^{\text {th }}$ centuries).

(m) Other likely Indo-European languages are Phrygian (Anatolian region), Messapic (Italy), Macedonian, Tarstessian and a number of other poorly attested ancient languages in Asia and Europe.

\subsection{The Six Phases of the Indo-Europeanization of the World}

We also propose the following Six Phases of the Indo-Europeanization of the World, even though some of these phases may be wholly outside the purview of our study.

(a) Phase A: The spread of various dialects and languages forming a part of the term Base Indo-European from the Indo European homeland and linguistic transformations in outlying regions would constitute what we may refer to as Phase A. These expansions would have normally been accompanied by human migrations which may have taken place not at one point in time as often erroneously assumed, but at different point in time to different outlying regions beginning from $3000 \mathrm{BC}$.

(b) Phase B: Billiard like extensions. This phase would comprise the Indo-Europeanization of Europe as explained in our papers, and the spread of Sanskrit within India as a liturgical language including parts of South India and its influence on Dravidian and other languages. This form of a spread may or may not have happened in tandem with human migrations. These may have occurred at much later periods of time in many cases and may constitute a form of elite dominance in many cases.

(c) Phase C: Tertiary extensions. This phase would comprise tertiary extensions like the spread of Sanskrit into South East Asia from India in pre-modern times.

(d) Phase D: This phase would comprise the spread of IE as a primary language over the past couple of centuries into regions such as North and South America and Australasia due to migrations from Europe.

(e) Phase E: This phase would comprise the spread of IE as a secondary language as a result of colonialism in places such as Africa and Asia.

(f) Phase F: This phase would comprise the spread of IE languages in modern times as a result of globalization. 


\subsection{Models Explaining the Indo-Europeanization of the World}

Several models of Indo-Europeanization have been proposed by different scholars. One of them is the Anatolian model proposed by Colin Renfrew which involves the spread of agriculturalists out of the Asia Minor region or Anatolia. One key and unanimous conclusion that has emanated from our previous papers on Indology is that the Anatolian hypothesis or the Anatolian Neolithic model proposed by Colin Renfrew and others (This became popular in the 1980's and has subsequently spawned a variety of versions) is untenable from India's point of view and be dropped forthwith in a wholly unmodified form, and subject of course, to other non-Indological considerations, if any. Renfrew's hypothesis which was modified several times over the past quarter of a century in response to strident criticism from Non-Indological and Indological scholars, initially sought to explain the spread of Indo-European languages into Southern Asia through the means of two possible approaches: a Plan A that saw the Neolithic economy spread south-eastwards from Anatolia to India (Thus, the Indus Valley Civilization was regarded as 'Indo-European' in this scenario. This is wrong because it requires pushing back dates to as far as $7000 \mathrm{BC}$, and was falsified on multiple grounds in our earlier papers; furthermore, the term 'Indo-European' is too ambiguous in today's parlance) or Plan B that explained the Indo-Iranians in terms of a much later migration of Bronze Age peoples from the Asiatic steppe lands southwards into the territory of southern Central Asia and the Indus. (From our perspective, parts of this approach may hold good. However, the actual solution to the problem is much more complex: readers may refer our earlier papers). Renfrew eventually abandoned Plan A for Plan B, however, a more recent solution to the homeland problem by Bouckaert et al. (2012), and also supported by Paul Heggarty, appears to argue for a variation of Renfrew's original Plan A, i.e., a homeland set in Anatolia in the Seventh Millennium BC with expansions both west into Europe and east into Asia. We seek to reiterate that all the models fail the complexities of Indian history very badly, and that author has the audacity and the temerity to state that these are, from India's perspective, herewith and summarily expunged.

The Near Eastern model is another model currently supported by a small minority of scholars. The chief proponents of this model have been the linguists Tamaz Gamkrelidze, Vyacheslav Ivanov and Stanislav Grigoriev. Per this model, the homeland is located south of the Caucasus, and Indo-European expansions are purported to have taken place somewhat later than the dates associated with the Anatolian Hypothesis (the spread of farming is not a critical element of the Near East model).

Marija Gimbutas' homeland which is a part of the much more widely-accepted Kurgan hypothesis or the Pontic- Caspian model, calls for a homeland including regions to the East of the Caspian Sea in the steppes and this includes Georgia, Azerbaijan and Armenia, and parts of Turkmenistan and Uzbekistan was well. Our theory will not cause any major disruption to this model, (as a matter of fact it tallies perfectly with all readings in Indology and must be recommended to scholars around the world) and our observations, upon a very preliminary analysis, call for extending the urheimat slightly eastward to include more regions in Turkmenistan, Uzbekistan, Tajikistan, Kyrgyzsthan, (encompassing Samarkhand, Dushanbe and Tashkent) and even parts of northern Afghanistan and regions to the west of the Khyber pass from where the purported migrations to India took place. Whether Anatolia will form a 
part of this homeland is open to debate. We do not know if the whole of the steppes will form a part of this postulated expanded homeland either. Modifications to Gimbutas' model have been proposed by scholars such as Kortlandt too, and this proposed a homeland in the Eastern part of Ukraine, or the north of the Caspian sea, and such wide divergences in opinion, which result in differences of up to three thousand kilometers or more in the source location, can die if a multi-PIE approach is indeed adopted. In the multi-PIE approach proposed in our previous papers, we stated that the PIE may not have been a single languages or just a group of dialects, but perhaps a language group in itself, spoken over a much larger spatial region than hitherto envisaged, and could also be envisaged as a space-time continuum of dialects or related languages. We can identify two stages. Stage A: Movements within the homeland after the domestication of the horse could have led to the dissemination of cultural traits and the evolution of common cultural traits within the Urheimat. (Alternatively, knowhow of horse domestication may have been passed on from community to community in the IE homeland, or a more realistic solution may have involved the combination of both scenarios) Stage B: Movements of people could have taken place from various parts of the Urheimat to Europe, Iran and India. We may note the following here: (a) it is not necessary that movements of people to Europe, Iran and India took place from the same location (b) it is not necessary that transhumance movements to Europe, Iran and India took place at the same time, and the differences in timeframes for migration for each of these regions may have been large, even if only one wave of migration to each such region had occurred. It is not even necessary that this language replaced all other languages in the outlying regions, but only interacted with them in a process of prolonged interplay as happened in the case of India. This is why we had even proposed the term 'Base Indo-European' instead of 'Proto Indo-European', which from our analysis, would not have been a single language, but a group of languages. This group would have comprised languages which disappeared within the homeland itself without impacting any other languages, and hypothetical extinct minority languages which may not have fitted into the term 'Base Indo-European' or 'Proto Indo-European'. These may be referred to as isolates.

Therefore, in an extreme case, the ideal solution may involve a partial combination of the Kurgan hypothesis the Anatolian hypothesis and the Near Eastern hypothesis with suitable modifications, implying that the homeland encompassed parts of all the three regions. Over the next couple of years, scholars can test whether such approaches work in the real world or not. Thus, knee-jerk reactions to new discoveries need to be avoided, and there is currently a tendency to change the location of the Urheimat as new data is found. Improved acculturation models from India can have a major bearing on Iranian studies and Indo-European studies in general, and we will be looking forward to such a revolution in the years to come. This can even unleash a transformation in the study of human languages and migratory patterns. This can be one of the key uses of the approaches presented in this paper, and they will thus be of immense scholarly value even to researchers who do not normally consider Ancient India to be a focus area of their study. This approach, of course does not superimpose pre-defined boundaries, but proposes various approaches that can be used or developed in the days to come to delineate and vet various future proposals for the boundaries of the Urheimat.

Therefore, we conclude that linguistic evidence would be the strongest in this case and can 
actually be considered to be the clinching evidence in a vast majority of cases. Archaeological evidence would come only a distant second, and the farming expansion model is open to very serious debate, and may not be tenable at all.

We consider the multi-PIE hypothesis (with the resultant group of languages known as Base Indo-European) to be one of the key premises of our approach and the potential key to seemingly irreconcilable contradictions. In other words, when the going gets tough for a scholar, the Urheimat is simply expanded to cover more geographical territory, or include more languages. To word it differently, our model supports the idea that the term 'Indo-European homeland' may have been a relatively vague idea in linguistic terms, a core Urheimat notwithstanding, and may have been more or less analogous to the region where horse domestication first took place. If contradictory evidence exists, the boundaries of the homeland need to be extended. (and not shifted!). This assumed added importance in view of the fact that J P Mallory and other leading scholars are unanimously of the opinion that no one model can explain all dimensions of the problem or even accommodate existing linguistic and archaeological evidence adequately. We will reiterate that gross over-simplifications are ill-suited to the realities of the real world. For example, the fate of the Neo-grammarians' supposed reconstruction of the PIE in the Nineteenth century is well-known. It failed an analysis of Hittite, which in this case may have largely a product of the local soil as well. Our approach is vastly different from the Space-time approach proposed by Meid as well.

Yet another possibility is a comparative analysis of all the languages in the Urheimat as identified from all the three approaches, and from our perspective, this can be a starting point of a study. This is one possible way - though perhaps overly complex, still very controversial at this point, and fraught with innumerable dangers and pitfalls en route. Methods to test this theory can be put in place, and this can include comparing aspects or features of present-day languages in the Urheimat which have already been overlaid by scores of other language groups not necessarily related to Indo-European e.g. Turkish (i.e. these languages need to be studied individually) with those of Europe and India, therefore necessitating some kind of a sub-stratum based approach, and tracing characteristics of languages in the Urheimat individually (we may refer to these as source languages from our perspective) to destination languages in Europe and India which will be Vedic Sanskrit, Old Avestan, Hittite, or other archaic languages of Europe. This approach can, in turn, be of major use in defining the boundaries of the Urheimat.

The obvious basis of this approach is of course the idea that only a small number of 'PIE speakers' would have left the homeland, and the same languages would have continued to be spoken in the homeland even after the migrations took place. However, this is much easier said and done or at least two reasons, even given the Author's rather minimalistic and rudimentary knowledge of the history and culture of Central Asia, and there will obviously be many glaring chinks in our armor: These issues, needless to say, are at the core of the complexity of the debate and intransigence of viewpoints. (a) The early languages in the Urheimat are not directly attestable and needless to say, there was no writing there at this point in time. (b) Language shifts have taken place in the Urheimat too, and these have been dramatic (research on the process and underlying causes has been poor): to Iranian, Turkish, 


\section{$\Lambda$ Macrothink}

Russian, Mandarin Chinese, and Even Arabic, and linguistic transformations there may have been extremely complex. There are a wide variety of languages spoken in the region, sometimes lumped conveniently into the controversial and ambiguous term 'Altaic'. Base Indo-European itself may have continued to exist in parts of the IE homeland until relatively recently as evidenced by Tocharian. (c) Innovative approaches such as sub-stratum based approaches as applied to the present-day languages of the region and identification of isolates in the region, along with work on other early cultures and civilizations of that area and a thorough study of the linguistic history of the region may help, although we may well be shooting in the dark at this point. (d) Time alone can bear witness to the outcomes of all endeavors, and whether at all such approaches are workable is unknown at this point (The author is somewhat skeptical himself)- They are certainly worth a try. If this does not work, the revised homeland can be delineated using some other methods described in our paper, and such approaches can be used as a stepping stone for more focused research. These two approaches may be called Approach A and Approach B from our perspective. If Approach A does not work, Approach B may still be eminently workable. In Approach B, the IE homeland needs to be worked out carefully using approaches not involving an attestation of the ancient languages of the homeland, but only indirect evidence, and may therefore include, partly or in whole, the Urheimat from all the three hypothesis i.e., the Kurgan hypothesis, the Anatolian hypothesis, and the Near-Eastern model. The earliest attested IE languages such as Vedic Sanskrit, Avestan, Ancient Greek and Hittite can be taken as a base and worked back using the approaches presented in our paper (We have describe ten different ways in which Indo-Europeanization or linguistic transformations could have taken place). In the case of India, this is certainly possible, and the ancestor of Vedic Sanskrit can easily be traced back to the language(s) of the Vedic homeland and earlier. This approach may help in the reconstruction of the "PIE" as a group of distinct inter-related languages.

Such models would also address another key point: motives for human migration. From the point of view of India, such migrations would not be hard to justify given the fact that small numbers of people were involved, and as a more recent study claims, migrants were males. The assertion that only a small number of migrants was involved has been made on various occasions by Asko Parpola, Witzel and Edwin Bryant as well, and would naturally stand to reason. A small group of males may have crossed the Khyber Pass accidentally on horseback, and may have been unable to find their way back, triggering a series of acculturations as was explained. A similar logic or reasoning may need to be worked out for other regions as well, and if the Urheimat were substantially expansive, migrations involving larger distances may not be warranted. Another factor to be always borne in mind is that these migrants were naturally more mobile than populations in surrounding outlying regions, given their association and natural ability with the domesticated horse.

Our observations from India also seem to indicate that a complete annihilation or replacement of languages cannot normally occur, expect in rare situations. Therefore, the other types of language replacement described in this paper would stand by us faithfully and in good stead in an overwhelming majority of practical situations. As Horton and Hunt have pointed out, assimilation and acculturation are always two-way processes involving some amount of give-and-take, and exceptions to this principle may be relatively rare. However, the Doctrine 
of Insubordination does imply that the subordinated languages are not on even keel with the subordinating languages, and as such it may take a greater effort to break their stranglehold. The relationship between the newly-discovered Jiroft Civilization and Old Avestan may also warrant further investigation taking into account our learnings from India and other parts of the world, and this is likely to push the boundaries of knowledge even further. Likewise, the languages of Europe may have not been linear descendants of the PIE as commonly assumed but may have only been heavily influenced by them or may have evolved as a result of a more complex interplay of linguistic forces. (However, the key difference in case of Europe is that Pre-Indo European cultures there may often have been less advanced than those of Iran or India). Such critical analyses may eventually beget improved acculturation models for Europe as well, if sensible use is made of scant data. We may refer to this as the Eighty Twenty rule, or one that would work in most situations. In other words, the observations made in our papers would hold true in at least eighty percent of situations. If any scholar wants to propose extremely simplistic scenarios (They may have got away with that in the past), they must back it up with adequate data and analysis. Another aspect to be borne in mind is a reconciliation of populations. We did that in India with amazing results and this must be likewise done elsewhere to avoid the dangers of over-simplification and erroneous conclusions. Likewise, several other erroneous and simplistic assumptions about human migrations and language replacement may need rethinking, and this exercise will undoubtedly push the boundaries of knowledge even further. As Michael Witzel states in his paper "The home of the Indo-Aryans", "Mallory's model is essentially a roller-ball model, an immigrant civilization joins the local one, transforms it by taking on many of its aspects, and then sets to move in a recurrent billiard-like fashion. "

\subsection{The Ten Golden Rules Associated with Human Migrations}

Therefore, before any model explaining the Indo-Europeanization of the world is formulated by any scholar in future, we must keep in mind the following (We will refer to these as the Ten Golden rules):

(a) The possible motives for human migrations which can be broadly classified into pull factors or push factors need to be studied on a case to case basis, they key assumption for our purpose being that humans need not migrate unless they need to do so. Even if this assumption does not always hold good in the real world, we will nonetheless insist on it, as it automatically places the onus on those proposing such migrations to carry out a basic introspection. This may render invalid any possible fantasies or over-simplifications like the crude Out-of-Africa theory.

(b) The possible motives for human migrations need to be studied much more critically when larger numbers of migrants are claimed.

(c) The possible motives for human migrations need to be studied much more critically when migrations involving larger geographical distances are claimed.

(d) The possible motives for human migrations need to be studied much more critically when migrations to regions with less salubrious weather conditions, or those unconducive to agricultural, economic or intellectual development are claimed. 
(e) The ability of the region in question to produce the numbers to facilitate migrations also needs to be taken into account, i.e. a reconciliation of the populations of the source location to the proposed size of the migrations needs to be carried out. By this we can avoid fantastic claims of large-scale migrations into India which are unsupported by genetic evidence.

(f) A reconciliation of the claimed size of migrations with the population of the destination likewise needs to be carried out. If this had been done in the case of India, we could have easily concluded that the number of migrants were small in relation to the Harappans, and that simplistic models of language replacement in this context would have been virtually impossible. For example, in the case of India, acculturation involving small groups of people can easily be explained from a 'Lost tribe hypothesis' - a small group of individuals accidentally crossed the Khyber Pass on horseback, and did not know how to get back, triggering a series of acculturations that was explained. It is expected that the number of migrants will be compared with the pre-existing populations of various destinations taking into account the estimated population of source destinations.

(g) The geographical features of the regions involved must always be kept in mind.

(h) It is expected that a reasonable quantum of additional direct and indirect evidence will be sought in support of any model to prevent an ideological misuse. This many include linguistic or archeological evidence.

(i) It is expected that this study is done in true letter and spirit and is used to test any model.

(j) It is expected that this study is done in true letter and spirit and is used to formulate 'Linguistic transformation models' for each destination separately.

\subsection{The Ten Types of Linguistic Transformations Associated with Human Migrations}

From our perspective, Linguistic transformations arising out of Human Migrations can be of the following ten types, and we briefly explain them below. Some concepts have already been put forth by J P Mallory, Witzel and other scholars, even though our compilation and classification of scenarios is much more comprehensive and detailed. These typically involve changes to two or more languages, and such changes can be of many types including word-borrowing, changes to grammatical structure etc. We propose the following ten scenarios of linguistic transformations in this context:

(a) Complete replacement or Annihilation: We had proposed in our earlier that this would be a very simplistic view, and one that may be practically rare and inapplicable to most situations. A scholar wanting to prove such a scenario, is expected to provide adequate data ruling out other scenarios. This is to preclude more complex analysis from being carried out. Such scenarios have however, happened in the real-world on occasion examples being the case of the USA and other parts of North America, where native languages were effectively annihilated. This can be justified on account of factors such as the nature or level of development of languages native to the region vis-à-vis those of immigrants, political factors, and the fact that populations may have been much more willing to assimilate in the emerging local culture as immigrants. The latter contributed to the linguistic suzerainty of English. In spite of this, French, another immigrant language, is only slowly dying out in Louisiana, and is far from dead in Quebec, where it is actually thriving, and is the chief language of the 


\section{MInstitute Macrothink $_{\text {Int }}$}

province. This process appears to have happened in Southern Sri Lanka as well, though in slow motion, as immigrants from North India in $600 \mathrm{BC}$ may have been relatively small in number. It is likely that other languages of the region, like those spoken by the present-day Veddahs of the region, were subsumed over a protracted span of time. However, as these languages may have also heavily influenced the language of the immigrants, and this may be a fit case for a roller-ball model, and not plain-vanilla or simplistic annihilation. The classification of this scenario as a roller-ball model may also be in order because the Sinhala language in its present form certainly did not exist when immigrations were purported to have taken place, and Sinhala is a product of Sri Lankan soil.

(b) Furthermore, other languages have not disappeared completely from Sri Lanka and languages other than Sinhala and Tamil are spoken in pockets including in areas where both Sinhala and Tamil are the majority languages. As this may be a protracted and long-drawn process in a majority of the cases, we may refer to it as the ripple-effect model as was observed in the case of Sri Lanka. This may also be referred to as the Survival of the fittest model as observed in the case of the USA. On the other hand, a dialect of English which was once spoken in the London area quickly became the standard variety of English. The death of other dialects of English was however only an extremely slow process only aided by factors such as urbanization, education, internal migrations and the popularity of English outside the UK. In much of the Middle East and North Africa, Modern standard Arabic is only used as a formal and a literary language, and the leveling of various dialects of Arabic appears to be nowhere in sight. Thus, Arabic is not only polycentric but also diaglossic. John Gumperz and Robert Wilson (1971) provide data from Kupwar, a small village in India, whose residents speak Marathi, Urdu and Kannada (A few also speak Telugu). These languages have co-existed for centuries without encroaching on, or destroying each other. This may be in part due to the fact that none of the languages in this case was dominant. All these examples should serve to illustrate that complete replacement or annihilation is not necessarily the de facto method of linguistic transformation. On the contrary, it may be relatively rare and needs to be justified or demonstrated though incontrovertible or relatively strong evidence. It is also a well-known fact that some languages of the Indo-European family are SVO languages, and some others are SOV languages. The process of change in the word order of languages (this itself may be relatively rare) may also be studied in detail so that linguistic transformations may be categorized appropriately. If the transformation of SVO languages into SOV languages or the other way around cannot be justified, we would have hit another linguistic jackpot: this would automatically throw light on the nature of linguistic transformation in different cases.

(a) Linguistic Sub-ordination: Linguistic Sub-ordination was another concept we had discussed in our previous paper, and may be relatively more common, at least in the short to medium term. We had also introduced the 'Doctrine of Insubordination' where we discussed that languages could cede functions to other languages much more easily, thereby losing them, rather than complete annihilation. This concept has been explained in detail in one of our previous papers. Allied concepts were 'Theory of Win-Win Propositions', 'Context and Role-based suitability', 'Context and Role-based indispensability'. Etc. 
(b) Roller-ball model (Single loop): In this case, an alien language spreads up to a certain point in a given region, loses its original form, and takes over characteristics and influences of a language or set of languages native to the region. The languages native to the region are also heavily influenced by the alien language, such that they are transformed significantly. Initially, both languages or language groups may continue to flourish for a while, as complete language replacement may be a much slower process.

(c) Roller-ball model (Double or Multiple loop): This is an extension of the Single Loop Roller-ball model where both groups of languages keep influencing each other as was observed in case of India. Refer our detailed model on the relationship between the PIE, Sanskrit and Prakrits which is also presented in this paper. Such complex scenarios may be relatively rare, and may have happened because 'Special conditions' such as de-synthesis and the spread back of 'Aryan' Culture into the North-West of India were involved. We have again presented a detailed account of how this may have happened in this paper.

(d) Extended Roller-ball model (Roller-ball model combined with Billiard-like extensions): In this model, a language spreads up to a certain point through one of the methods described above, and then spreads primarily through cultural diffusion at later points in time to various other outlying regions. This is what Witzel and J P Mallory may be referring to while using the term 'recurrent billiard-like fashion'. This may explain the Indo-Europeanization of most of Europe as Latin, already a member of the Indo-European language family, began to influence several other languages of Europe to give birth to languages such as French and Italian. This largely happened due to the power of the Roman Empire and several other political events which took place during and immediately after the fall of the Roman Empire. Latin heavily influenced Old English as late as the Tenth century, and French and even Scandinavian languages also similarly influenced Old English. However, this was unlikely to have been a direct or a simplistic replacement as presumed by most scholars. Most of the influences were through word borrowings, and it would be interesting to note that many Greek words entered languages like English through Latin. Additionally, Greek loan words were borrowed directly into English as well. This clearly demonstrates the complexity of factors causing or determining the inter-relationship between the world's languages, and we would again add a cautionary note urging scholars to desist from simplistic assumptions. The key evidence for a billiard-like of a spread comes from the wide variations in the dating of various languages belonging to the IE family, and this approach may be applied if the process of Indo-Europeanization in a given region began late. To study this methodically, a thorough knowledge of histories in various regions along with a knowledge of the Ancient and modern languages of the region is mandatory. This type of a scenario manifested itself in Ancient India too, with both Prakrits and Sanskrit influencing various Dravidian languages independently. Typically, the second type of a spread begins after the language has already achieved respectability in a given region or has established itself; examples here are Greek, Latin or Sanskrit. This explains how Sanskrit influenced the languages of South East also. A critical analysis of all our findings would of course rule out any notion that spread of languages necessarily happened in tandem with the movement of peoples. No other assumption can perhaps be as damaging to our cause than this. 
(e) Assimilation: The language(s) of the immigrants die out completely over a period of time, leaving behind virtually no trace. This is diametrically opposed to the idea of annihilation of languages, and may likewise happen under rare or special circumstances. Examples of such scenarios are Non-English speaking immigrants into the USA, and the decline of the Telugu language in Tamil Nadu. Persian in India did not survive long after the decline of the Mughal Empire in spite of the fact that it did impact the languages of the region.

(f) Cultural diffusion without transhumance movements or 'Pure Acculturation': This may be relatively rare from the perspective of Indo-European studies and the onus will necessarily be on proponents to explain how language replacement through either of the methods described above happened without any vested interests being involved. However, such paradigms are common in the Twenty-first century due to the ubiquity of technology. For example English has spread worldwide as a lingua franca without accompanying human migrations.

(g) Transhumance movements without linguistic change: In this scenario, there in minimal or no interaction between speakers at least in the short-term to the medium-term and both languages continue to exist in parallel. Such scenarios have been observed in India too, and may have been more common in case of pre-historic migrations. These were also a characteristic of language spread in colonial times.

(h) Lateral influences: Lateral influences are not just an interesting theoretical possibility: they have actually been observed in the context of Indo-European studies. I.e. lateral influences between Iran and India which played a key role in the evolution of Vedic Sanskrit. This possibility therefore needs to be considered at every stage in various contexts. Here, a layer-by-layer approach needs to be followed. For example, Hindi, which is a modern Indo-Aryan language was greatly influenced by Persian as well during the medieval era.

(i) Variants or combinations of the above scenarios may also be pervasive in the real-world and as such it is only expected that scholars will take cues from the above scenarios. Thus we wish to sidestep over-regimented approaches at every stage of the discussion.

We would refer to these as the 'Ten types of linguistic transformations associated with Human migrations.' In our view, and at least from our much more focused perspective which is centered on linguistic change wrought by transhumance movements, the above classification would constitute a vastly superior approach to existing classification models which are also discussed in passing in this paper, and should address linguistic changes arising out of transhumance movements at an enhanced level of granularity. This approach always talks about two or more streams of languages and adds several more dimensions the issue, including mandating a knowledge of local histories and issues. This approach can also provide supplementary explanations to vexatious issues such as the relationship of Hittite with other branches of the Indo-European family given the absence of several typical PIE features in this branch. JP Mallory has provided two plausible explanations, but our approach above can naturally provide more plausible explanations as whole-scale language replacement is not always the most credible scenario. As explained, our model supports the idea that the term 'Indo-European homeland' may have been a fuzzy idea in linguistic terms, and may have been more analogous to a region where horse domestication took place. Additionally, the rationale for the replacement of the term 'PIE' by the new proposed term 
'Base Indo-European' should be readily apparent to readers and we strongly believe this is the way ahead. We also strongly urge readers to be familiar with concepts like Role-based suitability, Context-based suitability, the theory of Linguistic Osmosis, The theory of Win-win propositions, the doctrine of Insubordination etc., which were presented in our previous papers.

Case studies and lessons learnt: It is expected that scholars will prepare cases studies and a list of lessons learnt for each case, the premise here being the each scenario can be different, and worth a detailed study, and no one-size-fits-all approach is possible.

\subsection{Other issues to be Considered}

The following issues would also be central to the Indo-European debate, and it is expected that a scholar would analyze them on a case to case basis.

(a) The number of immigrants vis-à-vis the numerical strength of the local population

(b) The technological and cultural superiority or attributes of the immigrants vis-à-vis the local population

(c) Cultural attributes and policy adopted by the local populations

(d) Attitudes or linguistic loyalties of the immigrants

(e) The Linguistic distance between the languages of the immigrants vis-à-vis the local languages

(f) The degree of linguistic inequality between the languages of the immigrants vis-à-vis the local languages. This will encompass sentence structure and aspects of Grammar too; for example we had used the fact the IA languages are SOV languages unlike most other IE languages which are SVO languages to campaign for a Roller-ball model.

(g) Other factors such as 'Linguistic Osmosis' (Refer to the 'Theory of Linguistic Osmosis' proposed in our previous paper) - This will include both extraneous factors and internal considerations such as ethnic rivalry etc.

(h) The level of evolution of the language of the immigrants

(i) The level of evolution of the languages of the destination area

(j) The standing of the immigrants in the social hierarchy of their newly adopted homeland.

(k) Any other factors or considerations understood from a detailed analysis of the issue in question.

Any discussion will be wholly void unless accompanied by a thorough knowledge of the local history or histories, culture, languages both ancient and modern, and knowledge of any other extraneous factors impacting such issues. A familiarity with linguistic and archaeological evidence pertaining to the area is also mandatory. Thus, we do not propose a model where a scholar is required to acquire proficiency in a large number of additional or distantly related languages in an unreasonably short span of time. For the author, or virtually anyone else in question, this would essentially be an unworkable proposition and a humongous waste of human resources. What is required is a collaborative effort between scholars of various hues and colors, across cultures and varying backgrounds, in such a way that the resultant synergies lead to an exponential increase in knowledge. We have many 
more examples to prove the importance of this kind of approach: for example, we had in our previous papers, demolished the idea of BMAC constituting archaeological evidence of migrations into India. We had also demolished the old Horse-chariot paradox, the Indo-Iranian language split-up, and such other myths, and this had worked wonders from our point of view. This is precisely what we refer to as fruits the 'Globalization of science' approach. Choosing the right resources is critical for the success of any endeavor such as this which is expected to be intensely multi-cultural, not just from the West and India, but from regions such as Eastern Europe, Iran, Turkey, Central Asia, Russia, China and South America as well, as this is expected to prevent the fossilization of intellectual cul-de-sacs and foster mutually beneficial dialogue, and Western scholars need to understand that India-based scholars may be ideologically-driven too, many of them incompetent.

The Indo-European language family comprises several main branches as was explained, and it is therefore expected that unique or appropriate techniques will be adopted for the study of each, will be adopted as required, keeping in mind the general principles above. These classifications were proposed by scholars such as August Schleicher, E. Sturtevant, Gamkrelidze, Ivanov, Vladimir Georgiev and Eric Hamp.

The 'Globalization of science' can yield many other rewards; instead of imposing or straitjacketing a Nineteenth century European worldview with all its foibles and infantile eccentricities, scholars from different parts of the world are expected to participate in all such complex endeavors: specialists from different regions in Eastern Europe, West-Central Asia and Iran, for example who are familiar with the different ancient and modern languages of their respective regions, those who are likewise familiar with the history and geography of those regions, and ideally those who are able to analyze world-wide problems in the light of problems pertaining to their regions, and challenge old or well-established ideas without losing sight of the big picture. In addition, it is expected that there will be centralized teams well-versed with theories of linguistic change and scenarios pertaining to the birth and death of languages. This will allow new light to be thrown on several old problems and allow fresh perspectives to manifest themselves in a way that will catapult knowledge to an altogether different league. These may even be in the form of completely new perspectives on old clichés or shibboleths.

Models proposed in the Mid-Nineteenth century may not have been just shaped by the zeitgeist of those times: they may have been shaped by the limited experiences of their proponents or experiences taken from their respective cultural backgrounds, just as Indian scholars' experiences may have been likewise extremely flawed or limited given their lack of training in scientific method or limited international exposure at that time. Firstly, Western scholars may have been overwhelmed by the linguistic and cultural diversity of the Indian sub-continent. Secondly, Western scholars have been conditioned by simplistic models of linguistic change as understood from an American perspective in the Eighteenth and Nineteenth centuries. Thirdly, Western scholars of the time may have lacked the crucial feel of Indian cultures at that time just as their Indian brethren may have had similarly narrow or limited views. Globalization in its present form is slowly but surely rendering concepts like 'National Character' obsolete. It is expected that the 'Globalization of Science' will ensure the process in logically completed by allowing experiences of scholars from different parts of 
the world to interact with each other meaningfully, leading to an exponential increase in knowledge.

The 'Theory of Linguistic Osmosis' that was explained in a previous paper, also has a bearing on language spread; English spread more or less in unmodified form across the globe because it was already a standard literary language at the time and because it already had currency elsewhere; these would not have been the case with any of the constituents of the PIE, allowing them to be modified or subsumed easily. However, in the case of early IE migrations from an IE homeland, 'Linguistic Osmosis' is of effectively no relevance.

\subsection{Older Theories of Linguistic Transformations}

Older models explaining the relationship of languages are now presented below for comparison:

The Tree Model: The Tree Model was first popularized by August Schleicher around the year 1860. In its simplest and crudest form, the 'Tree model' consists of a 'Proto-Language' (Proven or imagined), say 'A' branching into languages 'B', 'C' and 'D'. 'B', 'C' and 'D' in turn may have further branches. From our perspective, this antiquated model is too simplistic and theoretical to have any realistic value, at least in the context of Indo-European studies. Scholars would perhaps be hard-pressed to demonstrate real-world examples. The only valid exception perhaps that a language can give birth to a daughter language, but even here the forces shaping linguistic transformations can be extremely complex and varied.

Our criticisms of this model are therefore, many. These include:

(a) This model is too over-simplified to handle the complexities of the IE debate.

(b) Proto-languages cannot be assumed. This would be an erroneous assumption.

(c) Simplistic versions assume that the PIE was one language.

(d) It assumes over-simplistic models of language replacement. In real terms, the split of a mother language into two or more daughter language without wide-ranging influences from other languages or dialects may be relatively rare, or an extremely slow process. Even the evolution of Latin into French and Italian may have involved an interplay with a large number of other languages or dialects. We will recall the Eighty: twenty rule here. This involves a classification of scenarios into likely or unlikely. It is necessary for a scholar arguing for a scenario that may be unlikely to furnish additional evidence in support of his stand.

(e) Although the number of languages has oscillated through the ages, the general or the common tendency is for languages involving a small number of speakers to disappear and a consolidation of dialects to take place. Bona fide exceptions may be those involving migrations as these are triggers for linguistic change. The assumptions of the Tree model may render it unworkable in the real world in a vast majority of cases.

As Alexandre Francois has pointed out:

"Contrary to widespread belief, there is no reason to think that language diversification typically follows a tree-like pattern, consisting of a nested series of neat splits with loss of contact. Except for the odd case of language isolation or swift migration and dispersal, the normal situation is for language change to involve multiple events of diffusion across 
mutually intelligible idiolects in a network, typically distributed into conflicting isoglosses. Insofar as these events of language-internal diffusion are later reflected in descendant languages, the sort of language family they define - a 'linkage' - is one in which genealogical relations cannot be represented by a tree, but only by a diagram in which subgroups intersect."

As Heiner Fangerau, Hnas Geisler, Thorsten Halling and William Martin conclude in their book "Classification and Evolution in Biology, Linguistics and the History of Science",

"During the history of linguistics, the family tree never played a major role. Soon after the model was first introduced, scholars criticized the concept for its obvious shortcomings and proposed various other ways to model language history, none of which gained broad acceptance. The recent quantitative turn in historical linguistics which was initiated by the adaptation of new automatic methods initially designed for evolutionary biology led to an unexpected revival of the tree model in historical linguistics. Although the new methods doubtlessly decrease the amount of subjectivity inherent in the traditional intuitive approaches to phylogenetic reconstruction, they do not cope for the simplifying character of the tree model per se. In order to model language history in a realistic way, combining approaches which reflect the vertical as well as the horizontal aspects of language relations are needed."

The Wave Model

The Wave Model was first proposed by Hugo Schuchardt and Johannes Schmidt around 1870 as an alternative to the 'Tree model'. Under the Wave Model, an instance of language change arises usually from within a geographical region, and from there spreads to adjacent speaker groups. The propagation of the change is therefore like a 'wave' which expands away from its centre as the new feature is adopted by other languages usually in the region. Additionally, different features may spread independently of each other in different directions and different periods of time. According to Bloomfield (1933: 317), "Different linguistic changes may spread, like waves, over a speech-area, and each change may be carried out over a part of the area that does not coincide with the part covered by an earlier change."

The Wave Model, along with along its complex extensions, may not address all kinds of scenarios arising out of Human migrations, and is already ingrained as a part of one of the scenarios proposed in our paper.

Any approach will also need to address underlying causes of linguistic change over a period of time including Social Factors, Political factors, Technological factors, Cultural diffusion which may arise due to contact with aliens etc., and to similarly understand the principles of linguistic convergence and divergence. Our basic assumption here is that the rate of linguistic change is never constant and may fluctuate very widely due to a result in an interplay of all the above factors and as was amply demonstrated from our papers. It would also be necessary to understand such changes in conjunction with various factors so that linguistic changes can be better-justified or analyzed with underlying causes, and periods of relative non-linguistic change can be segregated from periods of relative linguistic change and similarly justified. 
1.8 Characteristics Associated with Different Types of Linguistic Transformations

The following are the key characteristics of the 'Ten different types of linguistic transformations' discussed by us in a previous section.

\begin{tabular}{|c|c|c|c|c|}
\hline S.No & $\begin{array}{l}\text { Type of linguistic } \\
\text { transformation }\end{array}$ & $\begin{array}{l}\text { Relative } \\
\text { frequency }\end{array}$ & $\begin{array}{l}\text { Status of component } \\
\text { of Base } \\
\text { Indo-European }\end{array}$ & $\begin{array}{l}\text { Effect on other } \\
\text { languages }\end{array}$ \\
\hline 1 & $\begin{array}{l}\text { Complete replacement } \\
\text { or Annihilation }\end{array}$ & Extremely rare & $\begin{array}{lr}\text { Base Indo-European } \\
\text { propagates } \\
\text { replaces } \\
\text { languages }\end{array}$ & $\begin{array}{l}\text { Decimation } \\
\text { annihilation }\end{array}$ \\
\hline 2 & $\begin{array}{l}\text { Linguistic } \\
\text { Sub-ordination }\end{array}$ & Common & $\begin{array}{l}\text { Base Indo-European } \\
\text { propagates }\end{array}$ & Sub-ordination \\
\hline 3 & $\begin{array}{l}\text { Roller-ball model } \\
\text { (Single loop) }\end{array}$ & Common & $\begin{array}{l}\text { Base Indo-European } \\
\text { transforms and is } \\
\text { transformed }\end{array}$ & Impacted \\
\hline 4 & $\begin{array}{l}\text { Roller-ball model } \\
\text { (Double or Multiple } \\
\text { loop) }\end{array}$ & $\begin{array}{l}\text { Under special } \\
\text { conditions } \\
\text { only }\end{array}$ & $\begin{array}{l}\text { Base Indo-European } \\
\text { transforms and is } \\
\text { transformed }\end{array}$ & Impacted \\
\hline 5 & $\begin{array}{l}\text { Extended Roller-ball } \\
\text { model (Roller-ball } \\
\text { model combined with } \\
\text { Billiard-like } \\
\text { extensions) }\end{array}$ & $\begin{array}{l}\text { Common } \\
\text { under a } \\
\text { specific set of } \\
\text { circumstances }\end{array}$ & $\begin{array}{l}\text { Base Indo-European } \\
\text { transforms and is } \\
\text { transformed }\end{array}$ & Impacted \\
\hline 6 & Assimilation & Common & Loses its identity & $\begin{array}{l}\text { Impacted } \\
\text { insignificantly }\end{array}$ \\
\hline 7 & $\begin{array}{l}\text { Cultural diffusion } \\
\text { without transhumance } \\
\text { movements or 'Pure } \\
\text { Acculturation' }\end{array}$ & Rare & $\begin{array}{l}\text { Base Indo-European } \\
\text { impacts other } \\
\text { languages and also } \\
\text { changes in the new } \\
\text { region }\end{array}$ & Impacted \\
\hline 8 & $\begin{array}{l}\text { Transhumance } \\
\text { movements without } \\
\text { linguistic change }\end{array}$ & Rare & $\begin{array}{l}\text { Does not impact } \\
\text { other languages and } \\
\text { is not impacted }\end{array}$ & No impact \\
\hline 9 & Lateral influences & $\begin{array}{l}\text { Under special } \\
\text { conditions } \\
\text { only }\end{array}$ & Not affected & Influenced \\
\hline 10 & $\begin{array}{l}\text { Various combinations } \\
\text { of the above scenarios }\end{array}$ & -- & As applicable & as applicable \\
\hline
\end{tabular}




\subsection{The Ten Dimensions of the Indo-European Debate}

Needless to say, the Indo-European debate has also been multi-faceted, and Harald Haarmann classifies issues associated with this debate into the following seven dimensions:

(a) The Economic dimension: This dimension deals with economic issues. For example, the transition from foraging to pastoralist subsistence.

(b) The Socio-Political dimension: For example, the emergence of stratified society and statehood in South Eastern Europe.

(c) The Ethnic dimension: this dimension deals with interaction between people of various ethnicities during the process of Indo-Europeanization.

(d) The Cultural dimension: this dimension deals with a fusion of different cultural traditions during the process of Indo-Europeanization.

(e) The Linguistic dimension: this dimension deals with language shifts arising due to the process of Indo-Europeanization. Here, a layer-by-layer approach needs to be followed. For example, Hindi, which is a modern Indo-Aryan language was greatly influenced by Persian as well during the medieval era. Again, spoken language needs to be differentiated from written language. We had observed that the spread of writing and the introduction of alphabets in Indo-Aryan languages in India followed a completely different path.

(f) The Visual-artistic dimension: The spread of visual imagery representing Indo-European traits forms a part of this dimension.

(g) The Mythical dimension: The study of Indo-European Gods and Goddesses forms a part of this dimension.

To this, we propose to add the following three dimensions:

(a) The Archaeological dimension: This dimension deals with all types of archaeological evidence associated with the spread of Indo-European

(b) The Technological dimension: This dimension deals the spread of technology such as the use of chariots and Iron in specific contexts - inevitably, both these technologies did not exist in the IE homeland.

(c) The Genetic dimension: This dimension deals with the Genetic evidence associated with the Indo-European problem.

We strongly emphasize that research on each of these dimensions must be carried out independently as there is little possibility of a complete overlap of issues associated with all the above dimensions. For example the spread of language may not happen in tandem with the spread of technology. Ignoring this vital issue would lead to an over-simplification of sorts and would greatly compromise the quality of research. Needless to say, this paper deals only with the linguistic problem and the linguistic evidence, and leaves out all other aspects of the issue to other specialists. Studying all dimensions of the problem will lead to an exponential increase in knowledge if the results from each dimension are analyzed cogently, without any other prejudice and with a view of furthering our understanding of the Indo-European problem. We can even refer to these as the 'Ten dimensions of the Indo-European Problem', and due credit must be given to Harald Haarmann for the first seven. 


\section{The Aryan Debate and the Origin and Meaning of the Term 'Aryan'}

There has possibly no term which has been as pervasively and persistently misused in recent human history as has been the term 'Aryan'; its misuse has been so widespread and rampant and has had such negative and highly objectionable consequences, that it would take much more than just an intellectual bulldozer to put things in proper perspective and undo what many would consider to be close to a century of mistruths and deceit. Research on the much-misused term 'Aryan' has been at the core of our research strategy, and a discussion is effected in the following few pages, with further logical and self-explanatory maps presented towards the end of the paper. At the very outset, it must be mentioned that the term 'Aryan' must be disassociated from the term 'Indo-European'. The reader must therefore, make a clear distinction between the two terms to avoid any further confusion.

In the early part of the Nineteenth century, scientists not only still believed in the now obsolete idea of human races, but also subordinated it to linguistic classifications. Furthermore, scholars such as Arthur de Gobineau proposed that races were unequal, and that the 'White race' was inherently superior to other races. He therefore claimed that the white race was responsible for key human achievements, and that racial intermingling usually had disastrous results often culminating in the downfall of great civilizations. The search for a nationalistic, Aryan centric national identity began after Germany's defeat in World War I in 1918. By the 1920's, the country was beginning to face a severe economic crisis due to the terms of the Treaty of Versailles of 1919. Hitler therefore strongly backed research on German pre-history to suit his party's ideology and misused the theories put forth by researchers such as Gustaf Kossinna to suit his own petty ends. One of his pet theories was that of a master and a superior 'Aryan race' which was used to describe people of European descent, or in some cases people speaking all Indo-European languages- this also owed to the fact that Indo-Iranian was known to be the most archaic form of Indo-European at that time. The idea of a superior Aryan race is repeated ad nauseum in Hitler's work 'Mein Kampf'. This approach of allowing racism to masquerade in the garb of science later came to be known as 'Scientific racism'. Under Hitler's definition, communities such as the Gypsies and the Slavs were considered inferior and the term 'Indo-European' was often loosely and recklessly used with the term 'Aryan'. As Marxist scholar late R.S Sharma himself concludes, "Towards the end of the eighteenth century, when William Jones discovered that Sanskrit was similar to Greek, Latin and other European languages, it was postulated that the Aryans lived in an area either in Central Asia or Eastern Europe. They were supposed to have descended from the same racial stock. This concept prevailed in the Nineteenth century and was used as a political weapon in Nazi Germany during the Anti-Jewish campaigns launched by Hitler. After 1933, it was declared that German people constituted a pure Aryan race. In the Nazi view, they occupied that highest place among Aryans and were hence entitled to hegemony all over the world. But scholars who have studied the Aryan problem deeply have come to the conclusion that those speaking the same language need not necessarily belong to the same racial or ethnic stock. Most scholars now think in terms of a proto-Indo-European language rather than once single race" (Sharma, 1999).

It is a well-known fact today that the term Aryan in India originated from the Sanskrit word 'Arya', meaning honorable, respectable or noble and that the actual origin of the term was 
probably Iranian. Acknowledging this, Max Muller stated "I have declared again and again that if I say Aryas, I mean neither blood nor bones, nor hair, nor skull; I mean simply those who speak an Aryan language. To me, an ethnologist who speaks of Aryan race, Aryan blood, Aryan eyes and hair, is as great a sinner as a linguist who speaks of a dolichocephalic dictionary or a brachycephalic grammar." Note: The term 'Aryan' was not used in a linguistic sense till modern times, and the term was at best cultural in the Rig Veda. We have discussed the meaning of the term 'Aryan' in great detail in the paper, "The demise of the Dravidian, Vedic and Paramunda Indus hypotheses: A brief explanation as to why these three hypotheses are no longer tenable", and key conclusions therefrom, are reproduced in this paper for the benefit of readers. As Edmund Leach states, "As Indo-European studies developed during the Nineteenth century, there was at first no fixed dogma about how the linguistic dispersion of the Indo-European family had come about. Around 1850, Max Muller was still vague. He wrote of the Aryan language, spoken in Asia by a small tribe, originally by a small family living under one and the same roof, as if it were the mother tongue of the human race. But prior to 1872 (and occasionally after that date) he was careful not to distinguish the study of languages from 'ethnology' the study of movement of peoples: 'There are Aryan and Semitic languages, but it is against all rules of logic to speak, without an express or an implied qualification, of an Aryan race, of Aryan blood, or Aryan skulls'. But the last quotation comes from a lecture given at the Imperial University of Strassburg in May 1872, just after the annexation of Germany by Alsace (against the express wishes of the local German-speaking inhabitants). In the earlier part of the lecture, Max Muller expressed his Germanic pride in the reunification of the German nation. His distinction between language and race is clearly under strain. By 1878, he writes without equivocation about the Aryans as if they were a group of people moving outward of some central Asian homeland by a process of conquest. By the 1890's, the linguist Sir George Grierson was explaining all Indo-Aryan dialect distributions in Northern India as due to past military conquest, a view that appeared without criticism in the 1969 printing of the Encyclopaedia Britannica. "(Leach 1990)

In the modern context, the term Indo-Aryan is most often used to refer to a group of languages forming a part of the Indo-European group of languages, whose discovery was famously announced by William Jones in Calcutta in 1786. Indus archaeologists also often loosely use the term 'Aryan' while referring to skeletal remains to differentiate them from other Harappan skeletal remains. This term is still misleading, however, as acculturation may have already taken place by then. As Witzel himself points out, attempts to look for physical Aryan bones or for an Aryan identity have generally proven unfruitful. While it is true that the late Dr. Gregory Possehl did talk about 'Aryan bones' at times, he, despite his pioneering work in Indus archaeology, in most other respects, and like living scholars like Asko Parpola and others, belonged, at the time of his death, to the now very antiquated Nineteenth century school of Indology. No one is perhaps more experienced in Harappan skeletal biology that Kenneth A R Kennedy. He has categorically and vehemently stated on more than one occasion that attempts to look for an Aryan biological entity have been unsuccessful.

Likewise, the Hungarian researcher Oswald Szemerenyi studied all the arguments pertaining to the term 'Aryan' and came to the conclusion that the term was not Indo-European but a near Eastern or an Ugaritic term meaning kinsman or companion. He concluded that there 
was very little evidence to show that the term 'Aryan' was used throughout the Indo-European world in ancient times. Scholars such as Gordon Childe, M.A. Mehendale, and Witzel have cautioned against erroneous interpretations of the term 'Aryan' but do not appear to have presented the breadth of meaning of the term 'Aryan' in their papers, or have succumbed to erroneous or half-baked interpretations themselves.

It would also be obvious to most people that the term 'Aryan' was used chiefly only in Ancient Iranian and Indian texts. In the context of the Indian Rig Veda, the term 'Aryan' means cultured or noble and does not connote ethnicity. However, in Iran, the term does appear to have an ethic connotation at times, and the term 'Aryan' appears to have been far more central to the Iranian identity than to the Indian Identity. Therefore, it would be obvious to most that the probability that it was imported from Iran to India was much, much greater than the other way around. In India, the homeland of the Aryans is called the Aryavarta, and corresponds to the Gangetic plains. The co-ordinates of the Aryavarta are clearly laid out in Sanskrit literature. Very interestingly, the term 'Aryavarta' in India does not refer to the Vedic homeland in the Punjab or a Central Asian Urheimat. This would again imply that the term 'Aryan' was not brought to India from Central Asia from where the migrations of IE speakers was purported to have taken place.

In Iran, the homeland of the Aryans is referred to as the Airyanem Vaejah. The location of the Airyanem Vaejah is also extremely controversial (Some scholars like Elton L.Daniel, Bahram Farah Vashi and Nasser Takmil Homayoun place it in Khwarazm in Central Asia while some other scholars place it in locations as far apart as regions immediately to the East of the Mediterranean and Afghanistan. In a few cases this includes places like Choresmia and Azerbaijan as well). The Avesta also lists some fifteen Aryan "countries". However, as the term 'Aryan' was used only in Ancient Iran and India, it is unlikely that the term Aryan was bought to Iran from an Urheimat. (If this had been the case, it would have spread throughout the IE world). Therefore, it was much more likely that the term 'Aryan' was coined in Iran where references to an ancient or a mythical homeland, the location of which was external to it, were added. The term 'Aryan' was therefore brought from Iran to India only during the compilation of the Rig Veda which took place between $1700 \mathrm{BC}$ and $1500 \mathrm{BC}$ due to cultural contacts with West Asia. This logic would hold good even if supposed Indo-European migrations into India took place much before the supposed Indo-European migrations into Iran.

The term Aryan, in reference to IE speakers in now obsolete, although a majority of Nineteenth century scholars like Arthur de Gobineau, George Vacher de Lapouge and Joseph Pomeroy Widney, among others, used it loosely and inaccurately in this context. In the Twenty-First Century, the term 'Aryan' is still often used inaccurately and in obsolete contexts only by very senior scholars and by the uneducated layman, although it would be illuminating to see how changes manifest themselves in the coming decades.

There is no known usage of the term 'Aryan' in the West before the eighteenth century. There are a few isolated references to this term by Western scholars. However, these occur in specific contexts only. For example, the Greek scholar Megasthenes only refers to 'Aranois' as people inhabiting the countries adjacent to India. It was only much more recently, in the Twentieth century, Hitler misused the ideas of Rosenberg, Houston Stewart Chamberlain and 
Gustav Kossinana to create the idea of a superior Aryan race, and this would be by far, the most extreme misuse of the term 'Aryan' that has occurred till date. Therefore, it would be evident, even upon a cursory and superficial observation, that the term 'Aryan', referring to a race or a group of people has its roots neither in history nor in science. [Trautmann, 1955, 20-30] As Witzel further points out "The combination of a specific language with any racial type is not maintained by linguists. At this late, post-Meso-Neolithic stage in human development, language no longer has any close relation to race. Recently developed methods of genetic testing will shed further light on this."

We now therefore arrive at the following conclusions:

(a) The idea that the term Aryan refers to a race is of course fallacious. The term does have an ethnic identity in Iran, (The usage of the term 'Aryan' in Iran is discussed elsewhere in this paper) but as the term 'Aryan' was coined in Iran, it could not have been synonymous with migrants who may have immigrated in earlier times. Admittedly, the term 'Aryan' sometimes does refer to more than one group of people in the RV, who were sometimes in opposition to each other, but the primary connotation of the term 'Aryan' in the RV is cultural.

(b) The idea that the term was used throughout the IE world in ancient times is also incorrect.

(c) The idea that the term always spread along with the movement of peoples is also incorrect.

(d) The idea that the term was brought to Iran from an Urheimat is also incorrect.

(e) The idea that the IE speakers to India (we proposed a date of $2750 \mathrm{BC}$ ) called themselves Aryans is similarly wrong. (In other words, the idea or notion that the term 'Aryan' was brought to India from an Urheimat is untenable)

We can, however, clearly establish the following:

(a) The term was coined in Iran and is fairly central to the Iranian identity, the country having been named after it.

(b) The geographical definition of the term Airyanem Vaejah was made in Iran, although, this location may itself have been outside of it.

(c) There is also a possibility as the term may have been brought from outside Iran as some scholars have suggested that the term was Ugaritic. The Ugaritic language was spoken in a region to the North-West of Iran. However, the term appears to have a strong ethic connotation only in Iran. It is also extremely unlikely, that the term 'Aryan' was used throughout the Urheimat for reasons already discussed. The history of the term 'Aryan' may be explored further, taking the work already done as a base.

(d) The term spread to India during the compilation of the RV phase in the Gangetic plains between 1700 and $1600 \mathrm{BC}$. This happened due to cultural contacts between West Asia and the Gangetic plains.

(e) Therefore, the term 'Aryan' in Ancient India clearly did not correspond with the word 'Brahmin' or with the IE speakers, who had lost their identity much, much earlier despite the possibility of an overlap.

(f) In ancient India, the term Aryan had the following five uses: 
(i) It mostly referred to a culture in the RV.

(ii) It was sometimes used in opposition to the term Dasas or the Harappans in the RV, but this term was always cultural and not racial (despite a small genetic input).

(iii) It was used in the term Aryavarta. The term Aryavarta, however, refers to the Gangetic plains and not the Vedic homeland or an Urheimat (which may have even been long forgotten by $1700 \mathrm{BC}$ or $1600 \mathrm{BC}$ ). This term appears in later Sanskrit literature only.

(iv) It was only in later Sanskrit literature that the term 'Aryan' acquired a linguistic identity, and was used in opposition to the term 'Mleccha' (this term is a derivative of Meluhha i.e. the Harappans)

(v) Even if the term 'Aryan' refers to more than one group of people in the RV, it was brought into use after several rounds of acculturation had occurred, and as the term was brought from Iran, did not equate to a small number of immigrants who may have migrated much earlier into India as well.

(vi) In the twenty-first century and beyond, the term 'Aryan' should not be used in a plural form. Likewise, the term 'Dravidian' should not be used in plural form either.

Unfortunately, obsolete paradigms continue to haunt us to this day, and Indology, in popular perception, has yet to be liberated from the ghosts of its past, such that the Indology taught in colleges and universities today is widely referred to as the Mid-Nineteenth century school of Indology, and is as such widely believed to be dying. This has prompted several calls for its resurrection in the recent past, both in Western and Indian circles.

As Thomas $\mathrm{R}$ Trautmann states, acknowledging the continuing legacy of colonialism "Speaking for myself, having been awakened from my dogmatic slumbers and having gone back to the British pioneers of Indology for a closer look, doing so has been a revelation for me, filled with unexpected discovery. At the outset, it became very clear that most the British Sanskritists, without any exception were empire loyalists and scholars who took it for granted that there was a very close connection between their scholarship and the British colonial adventure in India." (Trautmann, 1999).

From the Hindutva perspective, India had to be seen as the land of the 'Aryans', (a proposal we have extensively rubbished) as this would give the right for Hindus to claim to be the sons of the soil, allow inconvenient truths such as the caste system to be buried, and Christians and Muslims effectively demonized. According to Veer Savarkar, all Hindus were members of the Aryan race, and therefore not aliens. They were also united by the bonds of human blood, and descended from Vedic forefathers. According to him, a Hindu and an Indian was one for whom India was their pitrubhoomi and their punyabhumi. Little, however, do Hindutva ideologues realize that they themselves run the risk of using the term 'Aryan' in an ethnic or racial context through such misadventures. The Hindutva approach has little backing in the West excepting among a small group of Hindutva-inspired or Hindutva-sponsored writers. Apart from this, a few theosophists such as Colonel Olcott of the Nineteenth century had maintained that the 'Aryans' were indigenous to the Gangetic plains of Northern India.

Other vested interests too may have played a role in perpetuating antiquated myths. For example, Jyothiba Phule, who was a pro-Sudra and an anti-Brahmin writer, supported the idea of an Aryan invasion, and his writings had decided political overtones. Modern Dalit 
writers, and some Communists likewise may have a penchant for supporting antiquated paradigms, and this does not bode well for the future of science. As Romila Thapar points out, "In this version, the Aryan Invasion, was a necessary event and has now become an essential part of the Dalit version of history. It was to influence a variety of anti-Brahmin movements particularly in different parts of the peninsula, but more generally elsewhere as well. The dichotomy between Brahmin and non-Brahmin was seen to provide a rational explanation for the pattern of history and the suppression of the non-Brahmin. This dichotomy was easily slotted into the linguistic version of the Aryan and the Dravidian languages were Brahmins were Sanskrit-using Aryans and Dalits were seen as Dravidian speakers." We now know that this is a gross and an erroneous over-simplification, and all our readers will vouch for it as well. We must reiterate that science and objectivity are sacrosanct, and other ideologies must not be allowed to conflict with them in a way that will jeopardize objectivity or act against the interests of society. If this principle is adhered to at all times, we may be on the cusp of an exciting era of inter-disciplinary and inter-cultural research.

As Witzel further points out in his paper "Autochthonous Aryans? The Evidence from Old Indian and Iranian Texts":

"Scholars of the Nineteenth or the Twentieth centuries obviously did not have the present discussion in mind when they wrote. The best ones among them may have come to certain conclusions quite independently of their 'ideological' background. At any rate, the better scholars of the Nineteenth century were not colonialists or racists. They all were, however, limited to some extent by the general zeitgeist of the period, but so are present day scholars. We, too, must constantly strive to overcome this bias (Witzel 1999d), and we also must not to follow one current trend or momentary fashion after another. We can only approach a solution by patiently investigating the pros and contras of the various points that have been made -- or still are to be made. Scholarship is therefore an ongoing dialectical process. In the natural sciences and in scholarship at large, old conclusions are constantly reviewed on the basis of new evidence. But such new evidence has to fit in with the general framework established by the many, completely unrelated observations in the various branches of scholarship; otherwise a particular theory is revised or discarded. For example, when certain irregularities in the course of the planets were noticed, it did not mean that post-Renaissance astronomy was wrong but that this observation was due to the mass of another planet, Pluto that was correctly predicted and, then, actually discovered in the early 20th century. But, the opposite procedure, deducing a "paradigm shift" based on isolated facts, is quite common in the contemporary effort to rewrite Indian (pre-) history."

\section{Other Issues Associated with the Indo-European Question}

\subsection{The Usage of Maps and Diagrams to Show the Dispersal of Base Indo-European}

Maps are commonly drawn to show the spread of "Indo-Europeans", but these are far from satisfactory and seldom free from conceptual errors. In most cases, dates for dispersal, especially secondary movements are randomly or arbitrarily arrived at, and current models do not explain how linguistic transformations happened in outlying regions. Existing depictions also often lack detailed accompanying notes and often tend to leave readers completely nonplussed. This would naturally warrant some changes, and we present an approach below: 


\section{Macrothink}

We can use the following color scheme for enhanced clarity.

(1) The Red arrows will represent the constituents of Base Indo-European moving out of the homeland usually, though not always in conjunction with human migrations. Where dates are available, information will be provided alongside the arrows. In most cases, dates are expected to be approximations calculated in reverse from information available at the destinations.

(2) The Blue arrows will represent Billiard like extensions. These may be at several levels. For example, Sanskrit and Prakrits influenced Dravidian languages. Secondarily, Sanskrit may have spread to South-East Asia from South India at a later period of time.

(3) The Green arrows will represent tertiary extensions. Examples of these are the Indo-Europeanization of North America, South America and Australasia in modern times.

Different types of linguistic transformations may also be represented using different icons, and our proposals are as below. These will be useful while preparing diagrams and other kinds of visual depictions.

\begin{tabular}{|c|c|}
\hline S.No & Relevant icon \\
\hline 1 & $\begin{array}{l}\text { Complete replacement or } L \\
\text { Annihilation }\end{array}$ \\
\hline 2 & Linguistic Sub-ordination \\
\hline 3 & $\begin{array}{l}\text { Roller-ball model (Single } \infty \\
\text { loop) }\end{array}$ \\
\hline 4 & $\begin{array}{l}\text { Roller-ball model (Double } \infty \infty \\
\text { or Multiple loop) }\end{array}$ \\
\hline 5 & $\begin{array}{l}\text { Extended Roller-ball } \begin{array}{l}\text { Billiard-like extensions shown through distinct } \\
\text { model (Roller-ball model } \\
\text { arrows of the appropriate color }\end{array} \\
\text { combined with } \\
\text { Billiard-like extensions) }\end{array}$ \\
\hline 6 & Assimilation \\
\hline 7 & $\begin{array}{l}\text { Cultural diffusion without Arrows with dotted lines } \\
\text { transhumance movements } \\
\text { or 'Pure Acculturation' }\end{array}$ \\
\hline 8 & $\begin{array}{l}\text { Transhumance movements } \\
\text { without linguistic change }\end{array}$ \\
\hline
\end{tabular}




\section{IMacrothink}

\begin{tabular}{lll}
\hline 9 & Lateral influences & Arrows of the appropriate color \\
\hline 10 & $\begin{array}{l}\text { Various combinations of } \\
\text { the above scenarios }\end{array}$ & Uses the relevant icons sequentially \\
\hline 11 & Unresolved & $?$ \\
\hline
\end{tabular}

\subsection{Techniques to Identify Linguistic Transformations}

It is expected that scholars will work together to identify methods to study linguistic transformations and reconstruct the "Pre Indo-European" languages at various destination locations, and constituents of Base Indo-European in the IE homeland. A few examples of these can be as follows (However, it is early days yet, and this would serve the indicate the miraculous benefits of better and more focused research).

(a) Substratum-based approaches involving a study of the sub-stratum of transformed languages at the destination locations. For e.g. a formal of languages such as Greek or Sanskrit conducted to analyze their substratum. This is a traditional old-fashioned approach and has been in vogue since the early days of Indo-European studies and is subject to several limitations and pre-conditions which are discussed in this paper.

(b) Location-specific approaches. Readers are advised to go through the methods we proposed to reconstruct the languages of the Indus Valley Civilization. This would serve to illustrate to readers that there can be no substitute for location-specific expertise. This is exactly why the globalization of science as explained in this paper, assumes added importance.

(c) Approaches to work backwards from attested languages at destination location to make an attempt to identify characteristics of Base Indo-European. This can be done by identifying words and features that are known to be of local origin and eliminating them.

(d) Approaches to study the present day languages of the IE homeland in Central Asia, and work backwards or to look for isolates in the region. Whether this approach will succeed, is unknown at this point, as there have been major language shifts over the ages in Central Asia, as components of base Indo-European are not attestable in the Indo-European homeland, and have neither survived in the IE homeland in their original form. We will be looking forward to more research papers on controversial topics such as these, as we are still open to opprobrium and constructive criticism here.

Any other scholar-defined methods that may be developed by other scholars in future. It as valid in the field of linguistics to adopt different approaches i.e. travel up and down the linguistic tree and move backwards and forwards in time. It is expected that scholars will use such techniques to the fullest. If two streams, of languages are involved, it is expected that the above principles will be used to analyze changes to both streams of languages to the extent data is available.

\subsection{Changes in Approaches and Focus Areas}

Additionally, it is expected that there will be a major paradigm shifts in areas of focus, and we identify some of the below: 
(a) Identifying the IE homeland or Urheimat may no longer be the primary focus of Indo-European studies; and the boundaries of the homeland can be much more reliably be identified once the methods and approached presented in this paper are followed.

(b) The major focus going forward should be identifying and analyzing how linguistic transformations took place in various outlying regions and identifying as many new scenarios from across the world as possible.

(c) Another major focus should be identifying the characteristics of various components of Base Indo-European using the various approaches - or combinations of the various approaches - laid down in this paper.

(d) Another major paradigm shift would a globalized approach with scholars from many regions in the world participating, and the key principles for the above have been laid bare in this paper as well as our earlier ones.

(e) Another major paradigm shift would be multi-disciplinary approaches, and the key principles for this have been laid bare in our earlier papers.

\subsection{Additional Notes on Human Migration}

Human migration may be defined as the movement by people from one place to another with the intention of settling temporarily or permanently in the new location. The movement is typically over relatively long distances and from one country or region to another, but internal migration or migration within a country or region or shorter migrations of other kinds also constitutes human migration.

People migrate for a wide variety of reasons. They typically consider the advantages and disadvantages of staying in the same location versus migrating, as well as factors such as distance, travel costs, travel time, modes of transportation, terrain, and cultural barriers before arriving at a decision. Migration can be internal to a region or external. Migration is usually voluntary, but at times it may be involuntary or forced, or impelled in some other cases. In case of impelled migration, migrants are indirectly forced to leave a region due to unfavorable conditions. Migrations may be permanent or temporary. Seasonal migration is a type of temporary migration. Other types of migration are step migration or migrations executed in a series of small geographical movements, and chain migration which involves humans migrating one after another, usually from the same source and destination. Factors driving Human migrations may be classified into Push Factors and Pull factors.

Push factors include difficulties such as a food shortage, war, flood, etc. Pull Factors include those factors that cause people to move into a place because of something desirable such as a nicer climate, better food supply, freedom, desirability of the destination location etc. Several types of push and pull factors may influence people in their movements including:

1. Environmental (e.g., suitability of climate, frequent natural disasters)

2. Political (e.g., war, strife)

3. Economic (e.g., Employment related-issues)

4. Cultural (e.g., religious freedom, education)

Other factors such as the allure associated with a destination location may also form a part of pull factors that cause human migrations. 


\section{MInstitute Macrothink}

Ernst Ravenstein had proposed the following Laws of Migration way back in 1885:

1. Net migration amounts to a fraction of the gross migration.

2. The majority of migrants move a short distance.

3. Migrants who move longer distances tend to choose big-cities.

4. Urban residents are less migratory than inhabitants of rural areas.

5. Families are less likely to make international moves than young adults.

This interpretation is somewhat simplistic and outdated, and in this paper, we have laid down the factors that need to be borne in mind while analyzing human migrations. The identification and crystallization of laws may be construed as restrictive from our perspective, and the factors identified in our paper, as well as other scholar-defined factors may be analyzed to identify and put forth 'Principles of migration'. There may be no concrete laws, and for each principle, there may be a few bonafide exceptions- formulation of rigid laws may even be construed as an example of the over-simplification that has plagued the field, and other related fields for well over a century - indeed, in the real world, no two scenarios may be exactly alike, and this is one aspect scholars must always bear in mind.

According to the Gravity model, the interaction between two groups of people is proportional to the multiplication of the two populations divided by the distance between them; this phenomenon is distance decay (the effect of distance on cultural or spatial interactions). This interpretation too is extremely simplistic, and in this paper, we attempt to define the factors that determine the propagation of cultural traits under various situations. These, along with factors identified by other scholars are expected to replace such ageing constructs.

\subsection{Linguistic Transformation vs Migration of People}

It is often erroneously assumed that linguistic transformations are also accompanied by movements of peoples. While this is most often the case, there can be exceptions, and it must be admitted that these exceptions may be relatively few in number. There are some aspects that need to be kept in mind, and we list them down below:

1. Migrations would have at best involved small groups of peoples: This would most certainly have been the case with respect to the Indo-European question, because the populations of the Indo-European homeland, or any candidate for the Indo-European homeland, would have been relatively small in number, at least in comparison with their destination locations. It would also be very obvious that only a small proportion of residents of the homeland would have migrated to other locations.

2. Linguistic transformations may not have necessarily happened in tandem with movements of people, and for every rule, there may be countless exceptions. Linguistic transformations may have been of several types as explained in this paper.

3. Immigrants would not have called themselves 'Aryans'. The term 'Aryan' does not refer to a group of people migrating from place to place, neither does it equate to the migrants from the IE homeland or to the term Indo-European. The term may have originated in Iran, where it appears to have had an ethnic connotation; the term may have been coined either in Iran itself, or must have been imported, probably from an Ugaritic homeland or some other homeland (This itself lends credence to our Base Indo-European hypothesis or formerly, the 
Multi-PIE hypothesis.) The context of the term Aryan would have been primarily cultural in the Rig Veda, and its usage in a linguistic sense may not have manifested itself until much later. Its usage in the West, apart from its usage in restricted and specific contexts, was still more recent. Much of its recent usage in the west, and its usage to connate a race has been perverted, deceptive and fallacious. We have discussed the meaning of the term 'Aryan', which can no longer be used in a plural form in this day and age, at a great level of detail in this paper and our earlier ones.

4. Immigrants would not have called themselves 'Indo-Europeans', and the term Indo-European is of much more recent origin and does not equate with the term 'Aryan' either. In Twenty-first century scholarship, it would be unwise to impose modern terminologies on ancient peoples, without the use of qualifiers.

There has been an unhealthy and an unfortunate trend to mix up the two concepts, and while it is true that human migrations can usually be inferred from linguistic evidence more than any other form of evidence, observations and notes must ideally be made of human migrations separately in as far as much available data will allow.

\subsection{Linguistic Transformation vs Cultural Diffusion}

We believe that Cultural diffusion or diffusion of cultural traits is a wholly different concept, and must not usually be equated with either human migrations or linguistic transformations. Cultures may be defined as belief-systems whose characteristics are unique to a given society and typically include both social organization and technology. Change is an inherent part of any culture, and no culture can remain static in the long-term. There are many reasons for cultural change. Economic, political, environmental, technological, religious reform etc. in our case, migrations and contact with other cultures can bring about different kinds of Cultural change, and culture, in our context needs to be broken down into different cultural traits for a more detailed analysis. Many Cultural Anthropologists study languages in evolutionary or Darwinian terms and argue that the spread of cultural traits follow a process similar to Natural Selection.

Different cultural traits can propagate differently as was demonstrated in both parts of our paper, 'Syncretism and acculturation in Ancient India: A new Nine Phase Acculturation Model explaining the process of transfer of power from the Harappans to the Indo-Aryans' (This was renamed 'Syncretism and Acculturation in Ancient India: A new Nine Phase Acculturation Model explaining the transformation of Harappan India to Post-Harappan India' towards the end of Part Two of this paper and for very obvious reasons. Therefore, the origin of each cultural trait must be studied separately. In a given society the origin of a Cultural trait can be attributed to Source A, B, C etc. or to a combination of two or more sources. It may also be wholly or partly indigenous, and may have been transmitted from predecessor cultures. If transmitted from a predecessor culture, or from an external source, it may have been transmitted in modified or in wholly unmodified form.

The following factors would determine the propagation of cultural traits from a source location to a destination, and as such, must always be kept in mind while carrying out any analysis. 
(a) Availability at source: This is a very important factor to be bone in mind at all times. For example, we concluded that while Horse-riding in Post-Harappan India could be attributed to a Central Asian source, Chariot riding could not, and was instead traceable to Iran (or developed with knowhow from Iran or knowhow sourced via Iran.)

(b) Utility: The Utility of a trait (i.e. to what extent it satisfies a manifested, inherent or a latent need) would undoubtedly facilitate its rapid expansion, and in this case trade contacts with Iran led to enhanced equine trade.

(c) Appeal: The appeal of a trait may also aid in its propagation, even if has a relatively low level of utility. At times such a trait may even be harmful in nature, and yet its inherent allure could enable it to spread.

(d) Economy and practicability: The economy and practicability of a trait vis-à-vis other alternatives would play a key role in its propagation.

(e) Ethnic pride or Notions of Cultural superiority may play a role too in the dissemination of traits, and alien traits may be too often rejected under these circumstances.

(f) Authority: Cultural traits can also spread by virtue of political or religious authority.

(g) Availability of other alternatives that can satisfy a need too may play a role in determining the spread of cultural traits.

Different maps may be prepared, if required, to depict the spread of different cultural traits from location to location, and these would naturally not be synonymous with the spread, transformation or transmutation of languages in a vast majority of cases.

\subsection{Additional Notes on the Birth of Languages}

The origin of language has fascinated linguists and theorists since at least the beginning of the Nineteenth century. Even naturalists like Charles Darwin pondered over the origin of languages and called linguistic ability "an instinctive tendency to acquire an art." These is very little, if any, direct or indirect evidence, however, to theorize on the origin of spoken language despite the fact that some research has been done by scholars such as Alello and Dunbar, among others. Some scholars place the origin of spoken language at around 100,000 years BP, while other scholars place it at around 75,000 years BP. It is not even known whether spoken language originated in different parts of the world around the same time or at different periods in time, or whether spoken language originated at one place and then spread or whether it originated at different places independently. The author is personally supportive of the Multi-regional hypothesis of the origin of languages and believes spoken language may have evolved at different periods in time in different places in the world, and perhaps at vastly different periods in time to boot, given the fact that more than six thousand spoken languages belonging to many different language groups exist. However, the general idea of speech may have spread to neighboring regions in specific contexts, and in a slow process, due to various types of contacts, and would then have been adopted or modified as necessary to suit local conditions. Migrations would not have been necessary is a vast majority of scenarios to justify the spread of languages or the influence of one language on another. Therefore, theories of origin of language would also be largely independent of theories such as the now-contentious Out-of-Africa theory or any other rival theories, and looking for 
evidence of the origin of language in archaeological record would prove futile.

The birth of spoken languages would have been a result of a relatively high degree of cultural innovation. It would have also mirrored the social complexity of society, and would have been an epochal event in human history. Early linguists developed many theories to explain the origin of languages. Some of them are (a) The Bow-wow theory - proponents of this theories think languages originated from the sounds animals or other objects make symbolizing a kind of onomatopoeia (b) Pooh-pooh theory - According to this theory, languages come from responses people make to pain, surprise and other emotions.(c) The Ding-dong theory - according to this theory, language initially bore a resemblance with natural phenomena (d) The Yo-he-yo theory - According to this theory, speech arose from the chants and noises people made when they first worked with each other (e ) The Ta-ta theory According to this theory, speech came from the use of tongue and mouth gestures to mimic manual gestures. (f) The La-la theory - the theory that language came from music, lullabies and poetry. (g) The Wah-wah theory - according to this theory, language developed out of early cries of infants. (h) The Chew-chew theory- according to this theory, language evolved from oral gestures. Another approach categorizes the possible origin of language into (a) A divine source, implying speech was divinely inspired. (b) A natural source implying that language was a reflection of early sounds humans heard. (c) The Social interaction source which is similar to the Yo-he-yo theory. (d) The Physical adaptation source which implies humans mimicked sounds which were easy to pronounce. A theory similar to this is the meme theory. (e ) The Human intelligence theory states that humans invented language on account of their superior intelligence. In the recent past, Darwinian theories which see language as a part of the process of human evolution and adaptation have become very popular. More recent scholars such as Stephen Pinker have also called language instinctive. Some older theories may appear to be somewhat over-imaginative by today's standards. While parts of these may still hold good, future approaches should require that any assertions be backed up with empirical data.

Many words in a language may have been borrowed from neighboring cultures while many others may have been indigenous in origin. This would have been on account of at least two reasons (a) large number of word borrowings would not have been possible in such early times in the absence of any formal training or any written medium. (b) Each culture may have wished to demonstrate its own cultural identity, and nothing could have been more representative of this than language. Therefore, each culture would have had the need to differentiate its language from those of neighboring cultures. What may have been therefore witnessed in ancient times were dialect chains much like the spoken Arabic of present times.

Linguistic isoglosses may have been observed on account of physical or natural barriers and boundaries such as lakes, mountains and rivers. They may also have arisen due to political factors in many cases: for example, one tribe may have chosen not to get along with another tribe or had no cultural contacts with them.

\subsection{Linguistic Change: Consolidation of Dialects and Ethnogenesis}

Consolidation of dialects into languages or dialect levelling may have taken place due to many factors, the chief of them probably being political: as clans coalesced into confederates and proto-kingdoms in due course, the process of standardization of languages may have 
accelerated. This would have also led to the creation of isoglosses. The second factor driving the standardization of languages may have the emergence or writing and the wider dissemination of writing and this would have been a vehicle for enforcing political writ. Thirdly, natural factors such as the presence of mountain ranges would have also enabled the creation of linguistic isoglosses. Conversely, ethnogenesis or the breakup of political units may have triggered the birth of new dialects or languages, even though such scenarios may be relatively rare in modern times. They may have been more common in ancient times when communication systems were more primitive. Even then, the birth of new languages may have been a relatively slow process and may have been triggered by special conditions. Even though the number of languages has oscillated through the ages, the number of languages is steadily on the decline, leading further credence to the hypothesis that the factors leading to a decline in the number of languages outweigh the factors leading to the birth of new languages. However, the death of languages may at best be a slow process in a vast majority of situations as discussed in our pervious papers. Languages may lose some of the function or may be sub-ordinated, but may not die out easily, except in specific scenarios. Even when they do die out, the process is likely to be a long-drawn out one, preceded by the loss of the language's prestige and competence of speakers. The birth of American English or Quebecois French may be cited as examples for the emergence of a new dialect, although their development may have been modulated by what we referred to as the 'Theory of linguistic Osmosis'. We may be hard-pressed to document the birth of wholly independent languages in modern times. Likewise, the idea of a real or an imaginary proto-language mechanistically breaking up to form new languages (Tree model) may be a naïve or an outmoded assumption.

\subsection{Formation of Language Groups}

Language groups may have been the result of more permanent natural barriers such a mountains, large rivers, seas and oceans which may have led to a separation between two linguistic groups. In some other cases, they may have been a result of extraneous factors such as human migrations leading to linguistic transformations in a given region. Scholars are well-advised to study the formation of language groups more closely taking into account the various forms of evidence discussed in this paper, as well as political and historical factors. These may prove to be veritable jackpots and could yield rich cues on the origin and spread of languages.

\subsection{Proto-languages and Language Groups}

The idea of a hypothetical proto-language being an ancestor of even a bona fide family of languages, likewise, may be an extreme over-simplification by today's standards and may be a legacy of the antiquated tree model. We therefore choose to add a cautionary note here, as such erroneous and over-simplified conclusions create more chaos and confusion in the longer term, undermining progress on critical and crucial fronts, and such chestnuts may at best be avoided and permanently consigned to history. Alternative explanations may be sought in such cases, as far as they are apparent, as the idea of a language splitting up into many different languages without any apparent underlying reason, seems not only highly over-simplistic, but also inherently flawed. Some early scholars believed that all the world's languages could be traced to a handful of proto-languages. Creationist Carl Wieland once 


\section{Macrothink}

remarked: 'The evidence is wonderfully consistent with the notion that a small number of languages, separately created at Babel, has diversified into the huge variety of languages we have today.' This statement would of course constitute a gross over-simplification to anyone who has read the concepts in our paper and would even appear somewhat ludicrous. Relationships between languages whether forming a part of a bona fide family of languages or not, were perhaps wrought in many different ways, and some scenarios have been analyzed in our paper. We now analyze a few of the world's so-called proto-languages. A more detailed analysis may be warranted in each such case, and such an analysis should serve to demolish the myth of proto-languages in an overwhelming majority of cases:

\begin{tabular}{|c|c|c|}
\hline S.No & $\begin{array}{l}\text { Name of the } \\
\text { "Proto" Language }\end{array}$ & Additional notes or Alternative explanations \\
\hline 1 & $\begin{array}{l}\text { Proto } \\
\text { Indo-European }\end{array}$ & $\begin{array}{l}\text { We had proposed that this term be replaced by the term Base } \\
\text { Indo-European for two reasons (a) Base Indo European may } \\
\text { have constituted more than one dialect or language (c) The } \\
\text { constituents of Base Indo-European may not have necessarily } \\
\text { replaced languages in outlying regions but may have influenced } \\
\text { them in various ways as explained in this paper. Anyone who } \\
\text { has read and grasped our model of linguistic transformation will } \\
\text { unequivocally conclude that there can be no single family of } \\
\text { languages with the appellation 'Indo-European' and that this } \\
\text { term may at best be hackneyed. Different constituents of Base } \\
\text { Indo-European may have influenced various languages of } \\
\text { different regions in different ways. However, the term } \\
\text { Indo-Aryan may represent languages that technically constitute } \\
\text { a language family. }\end{array}$ \\
\hline 2 & Proto-Dravidian & $\begin{array}{l}\text { This concept has not scientific basis whatsoever, and } \\
\text { Proto-Dravidian may never have been one single language. } \\
\text { Instead dialects which may have instead formed a continuum } \\
\text { may have later crystallized into languages due to various factors } \\
\text { which are discussed in this paper. }\end{array}$ \\
\hline
\end{tabular}

3 Proto-Afroasiatic Such a language may not have existed. Migrations cannot or Hamito-Semitic naturally be invented to support theories formulated on such shaky grounds. The Elmo-Dravidian hypothesis is another non-starter.

4 Proto-Romance The hypothetical ancestor of the Romance family of languages. It is doubtful if such a language existed at all.

5 Proto-Greek Refers to the hypothetical ancestor of various dialects of Greek. The reader can apply the concepts in this paper to evaluate whether such a language existed in reality.

6 Proto-Anatolian The reader can apply the concepts in this paper to evaluate 


\begin{tabular}{lll}
\hline & whether such a language existed in reality. \\
\hline 7 & Proto-Balto-Slavic & $\begin{array}{l}\text { The reader can apply the concepts in this paper to evaluate } \\
\text { whether such a language existed in reality. }\end{array}$ \\
\hline 8 & Malayo-Polynesian & $\begin{array}{l}\text { Another questionable language family, the existence of which } \\
\text { needs to be revisited in the light of our observations. }\end{array}$ \\
\hline 9 & Proto-Tocharian & $\begin{array}{l}\text { The reader can apply the concepts in this paper to evaluate } \\
\text { whether such a language existed in reality. }\end{array}$ \\
\hline 10 & Elamo-Dravidian & $\begin{array}{l}\text { This concept, originally proposed by David McAlpin, is now } \\
\text { largely considered to be outmoded }\end{array}$ \\
\hline
\end{tabular}

\subsection{Additional Notes on the Death of Languages}

Languages can indeed die out, but scholars need to bear in mind the fact that this is typically a slow process: this aspect needs to be borne in mind while studying linguistic transformations arising due to human migrations as well. Language extinction can therefore only occur when the language has no competent language speakers and when the language falls into disuse. This naturally would be a slow process. Exceptions to this, if any, must be justified adequately by scholars. In this connection, we may draw the reader's attention to Walsh's four stages of linguistic decline which are:

(a) Language shift or language decay is a category of language decline where speakers have a limited vocabulary in their native language and more often use a new language in which they may be semi-fluent or fluent. (Hill, 2001)

(b) Language endangerment exists when a language has fewer than 10,000 speakers.

(c) Near-extinction is a situation in which only a few elderly speakers are still living.

As Salikoko F. Mufwene of the University of Chicago states, "Likewise, languages cannot be issued death certificates either, not really the kind that can be issued for an organism, a human being for instance. Although we have usually claimed that a language dies when its last speaker is dead, reality also tells us that the process of death itself started long before the death of the last speaker (Thomason, 2005), when the population of its speakers lost their critical mass and often also when its structures were seriously eroded by those of the prevailing language, as in the case of Sutherland Gaelic (Dorian, 1981)."

Needless to say, these observations would be crucial in formulating comprehensive perspectives of linguistic change associated with Indo-European studies, as we have always spoken of a pair of languages associated with any linguistic transformation.

\subsection{Towards Comprehensive Perspectives on Linguistic Change}

This paper only covers linguistic changes arising out of Human migrations. For a more comprehensive perspective to be obtained, linguistic changes arising out of other scenarios also need to be taken into account, although we will leave this to other scholars. Examples of the latter have also been discussed in passing in our paper. 


\section{Macrothink}

Therefore, from our perspective, linguistic change or linguistic transformations can be of two types:

(a) Linguistic change or linguistic transformation arising due to human migrations

(b) Linguistic change or linguistic transformation not arising due to human migrations

The former has been covered in a respectable level of detail in this paper and would represent changes wrought through extraneous means. However, we emphasize then two scenarios can almost never be the same, and even if great effort is put into compartmentalization of real-world experiences into different types of scenarios, there are always bound to be subtle differences in each case. We have therefore called for the 'Globalization of Science' and have encouraged scholars from different regions of the world familiar with their respective regions to identify new scenarios and variations to such scenarios and themes. We cannot and will not make any attempt to boil the ocean here. As comparativists and synthesizers we need to know our limits; the latter i.e. linguistic change not arising due to human migrations, needs to be studied at a greater level of detail by other scholars or linguists not specializing in the spread of IE. The two, when taken together, would constitute of proposed replacement for the Tree model and the Wave model. 


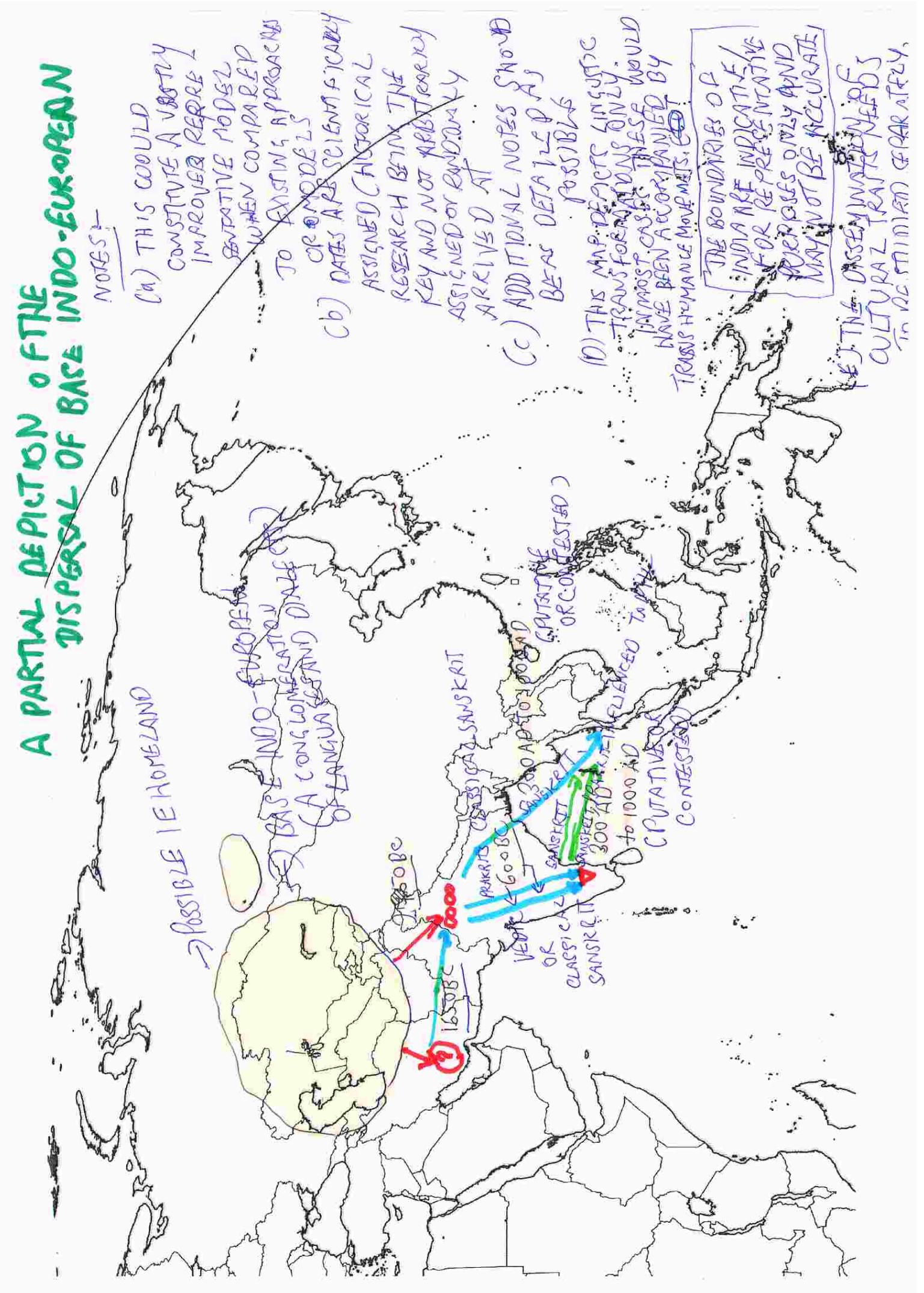




\section{Macrothink}

Journal of Social Science Studies

ISSN 2329-9150

2016, Vol. 3, No. 1

3.13 Additional Notes on the Diagrammatic Representation 'A Partial Depiction of the Dispersal of Base Indo-European'

(a) Our approach should provide a vastly improved and a much more scientific representation of the Indo-Europeanization of the world over existing models.

(b) A key concomitant of this approach is better historical research and research of this kind must necessarily include region-specific teams with a thorough knowledge of the local history, culture and conditions.

(c) This approach would therefore provide accurate dates instead of randomly-assigned dates. This approach may throw up dates ranging from as early as $3000 \mathrm{BC}$ to $1800 \mathrm{AD}$ accounting for the spread of languages like English, French and Spanish to North America, South America and Australia and other colonies as a second language. In the post-globalized scenario, the date range may be extended to cover the spread of English as a result of Globalization.

(d) This approach therefore emphasizes 'Globalization of science' which would in turn lead to 'Scientific progress at the speed of light'.

(e) Base Indo-European would have comprised of multiple dialects and languages and not just one language.

(f) Linguistic transformations would have been of at least ten different types as explained in this paper. The expression 'Linguistic transformations' is more appropriate than 'Language replacement'.

(g) Information on possible additional types of linguistic transformations can be gathered by interviewing people from different parts of the world as is explained in our papers.

(h) This paper lays great emphasis on the fact that two scenarios can almost never be exactly the same in real-world situations.

(i) Note the color scheme used for arrows. This comprises red, blue and green arrows as was explained elsewhere in the paper.

(j) Note the different types of icons used for different types of linguistic transformations. If in doubt, a question mark or an encircled question mark should be used.

(k) The depiction is expected to be as comprehensive as possible. For example we have shown how both Sanskrit and Prakrits impacted Dravidian languages.

(1) Additional notes must be provided as necessary so that a balanced picture is communicated to readers. For example, we have discussed that some scenarios especially those pertaining to the Sanskritization of South-East Asia were putative.

(m) Some creativity is always expected of a scholar. For example, the double loop shown in the case of India may be replaced by a double loop followed by an equal to sign as both Sanskrit and Prakrits continued to exist in parallel in Post-Harappan times.

(n) There can be no substitute for plain common sense. For example, spoken language needs to be differentiated from written language. We had observed that the spread of writing and the introduction of alphabets in Indo-Aryan languages in India followed a completely different path. 
(o) Lateral influences would have been fairly complex. For example, Avestan influenced Vedic Sanskrit. Again, Persian influenced Modern Hindi in recent times.

(p) It may be necessary to break up such maps by period in the interests of clarity; we have shown, for example, how complex and multi-layered influences of Latin, Greek, French and other languages were on English. A great deal of clutter may thus be eliminated if maps are represented by period wherever necessary.

\subsection{The 'Globalization of Science' and the Need for an International Approach}

We have spoken extensively about the 'Globalization of science', as we feel it holds the key to better long-term progress. There can be many different ways this can be put to work. In the proposal presented below, there will be three teams:

(a) Region-specific teams: This team will comprise of region-specific teams, and it is likely that there will be several such teams in place representing each region. The key pre-requisite here will be a thorough knowledge of local histories, conditions, culture and languages. Ideology-driven approaches will be strongly discouraged and it is expected that members of this team possess a scientific mindset and a fair modicum of competence in all allied areas too. However, nationalistic approaches are a strict no and must be eschewed always. This team may comprise of different sub-teams representing different specializations. Members of region-specific teams are expected in interact on a regular basis with members of the core or administrative team.

(b) The Core or administrative team is expected to provide strategic inputs to various teams and also carry out administrative functions. It is expected to liaise and co-ordinate between the various teams as well.

(c) Specialized teams will comprise of domain-specific experts and may be brought in as required. Examples of such domains are archaeology, linguistics and genetics.
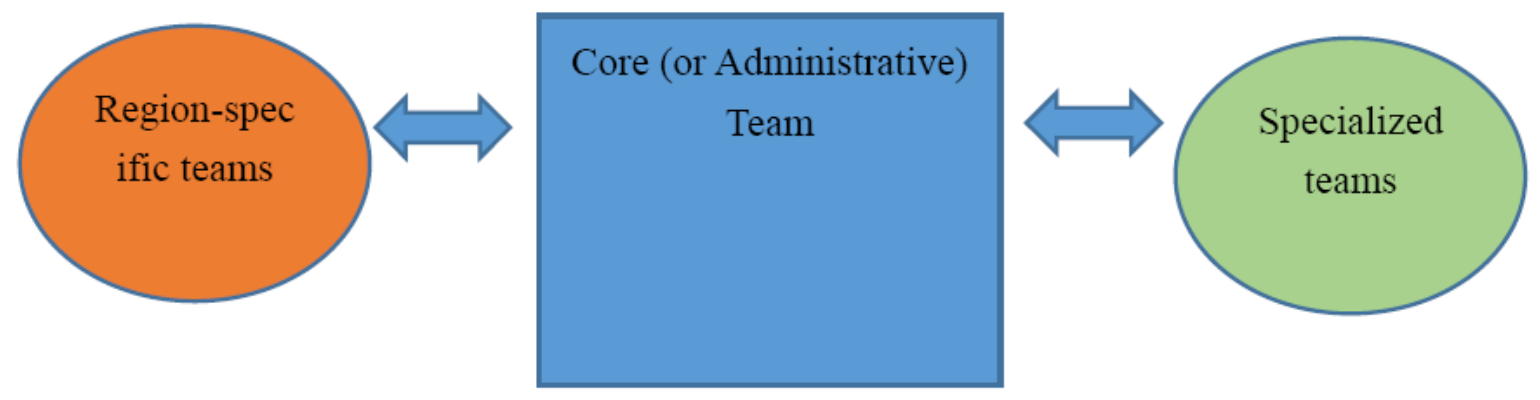

Examples of region-specific teams are given below. Countries could again be classified into Groups, and focus areas could be likewise, identified to provide a clear sense of direction. The Primary group would comprise of areas where Base Indo-European was originally spoken, the Secondary group would comprise outlying regions i.e., where the linguistic transformations were purported to have taken place. The tertiary group would represent countries which came under the linguistic ambit of IE at a much later point in time. 'Other' would refer to residual countries. 


\begin{tabular}{|c|c|c|c|c|c|}
\hline \multirow[b]{2}{*}{ S.no } & \multirow[b]{2}{*}{$\begin{array}{l}\text { Country } \\
\text { Group }\end{array}$} & \multirow[b]{2}{*}{ Name of Country } & \multirow[b]{2}{*}{ S.no } & \multicolumn{2}{|c|}{$\begin{array}{r}\text { Journal of Social Science Studies } \\
\text { ISSN 2329-9150 } \\
2016 \text {, Vol. 3, No. } 1\end{array}$} \\
\hline & & & & Group & $\begin{array}{l}\text { Name of } \\
\text { Country }\end{array}$ \\
\hline 1 & Primary & Kazakhastan & 9 & Secondary & $\begin{array}{l}\text { India (focus } \\
\text { area) }\end{array}$ \\
\hline 2 & Primary & Azerbaijan & 10 & Secondary & $\begin{array}{l}\text { Pakistan } \\
\text { (focus area) }\end{array}$ \\
\hline 3 & Secondary & Latvia & 11 & Tertiary & Australia \\
\hline 4 & Secondary & Lithuania & 12 & Tertiary & New Zealand \\
\hline 5 & Secondary & Estonia & 13 & Tertiary & USA \\
\hline 6 & Secondary & Spain & 14 & Tertiary & Argentina \\
\hline 7 & Secondary & Greece (focus area) & 15. & Tertiary & Canada \\
\hline 8 & Secondary & Iran (focus area) & 16. & Other & Yemen \\
\hline
\end{tabular}

\subsection{Summary of Lessons Learnt}

The summary of lesson learnt from region-specific teams may then be tabulated as below, and further conclusions drawn. This can then be used to identify newer scenarios which will need to be reviewed by an external party and will then form the basis of further analysis in global contexts.

\begin{tabular}{llllll}
\hline $\begin{array}{l}\text { Sno } \\
\text { Date }\end{array}$ & $\begin{array}{l}\text { Name of } \\
\text { the } \\
\text { Proposer }\end{array}$ & $\begin{array}{l}\text { Name of the } \\
\text { country }\end{array}$ & $\begin{array}{l}\text { Details } \\
\text { proposal }\end{array}$ & $\begin{array}{l}\text { of } \\
\text { reviewer }\end{array}$ & $\begin{array}{l}\text { of } \\
\text { conclusion }\end{array}$ \\
\hline 1 & $<>$ & Sudan & $<$ & $<$ & $<$ \\
\hline 2 & $<>$ & Cambodia & $<>$ & $<>$ & $<>$ \\
\hline 3 & $<>$ & Vietnam & $<>$ & $<$ & $<$ \\
\hline 4 & $<>$ & Bulgaria & $<>$ & $<$ & $<$ \\
\hline 5 & $<>$ & Australia & $<>$ & $<$ & $<$ \\
\hline
\end{tabular}

\subsection{The Transformation of Base Indo-European in India}

In this section, we will show how the interplay between the two streams of languages took place leading to the transformation of a component of Base Indo-European in India. This will be self-explanatory once the maps in a different section of this paper are understood, and would constitute what we referred to as a double-loop type of linguistic transformation. The objective of this section is therefore, to furnish an example of this type of linguistic transformation so that it may be useful to scholars in other parts of the world. We had proposed that the Indus Valley civilization was intensely multi-lingual, and its inhabitants 
spoke many languages which included remote ancestors of languages which much later came to be known as Prakrits. This hypothesis is supported in some form by Madhav Deshpande, Ahmad Hasan Dani, S Kalyanaraman and several others as discussed in our earlier papers. Speakers of the PIE or a constituent of Base Indo-European would have settled in the Vedic homeland in 2750 BC. Sanskrit spread into the Gangetic plains when these people moved to the Gangetic plains in 2300 BC-2200 BC. It died out as a spoken language when the eastward movement of the Harappans took place in 1900 BC, and became a liturgical language, and popular as a lingua franca of the elite. The speakers of IE languages then took on the languages of the descendants of the Indus for everyday speech because of the transfer of populations to the Ganga-Yamuna doab, and these later evolved into various Prakrits. Sanskrit then re-influenced the languages of the region, in a process that continues to this day even after it disappeared as a spoken language. For further clarity, readers are advised to read our previous publications on the Aryan problem as these explain how the below-mentioned model was arrived at in vivid detail.

The transition of the PIE or one of the constituents of Base Indo-European would therefore have taken place as follows:

PIE or constituent of Base Indo-European (before 2750 BC) $=>$ Some Ancestor of Vedic Sanskrit in the Vedic homeland (which could have influenced and been influenced by the languages of the region) (between 2750 and 2300 BC) => Again influenced by the early languages of the Gangetic plains before 1900 BC when the Indo-Aryans spread beyond the Vedic homeland (2300 BC to 1900 BC) => Was influenced by the languages of the IVC after the eastward movement of the Harappans in 1900 BC and also influenced them (1900 BC) $=>$ killed itself $(1900$ to $1600 \mathrm{BC})=>$ Became a liturgical language $(1900$ to $1600 \mathrm{BC})=>$ Also became a lingua franca of the upper echelons of society (1900 to 1600 BC) $=>$ This language took on influences from West Asia $(1700$ to $1400 \mathrm{BC})=>$ (The substratum in Sanskrit would consist of all the above influences) $=>$ This liturgical language re-influenced the other languages of the region in several periods, right up to the modern period after it became a symbol of Indian culture. For example, in the first instance, the competition between Buddhism and the threatened Vedic faith appears to have seen the re-emergence of Classical Sanskrit. In the second instance, the religion that we nowadays refer to as Hinduism appears to have become popular in the 10th Century. There have been movements promoting the Sanskritization of Indian languages even in recent times.

The transformation of the now extinct languages of the IVC had happened as below:

Ancestors of Semitic (some Ancient period around 7000 BC, perhaps) => Some early form of Indic after $3300 \mathrm{BC}$ and $2600 \mathrm{BC}$ due to (however, there would have been a vast difference between the first two because of the vast time gap and it is natural to expect that there would have been several types of people living in the North-west of India and Pakistan already). The $I V C$ itself was perhaps polyglot $=>$ Heavily influenced by Sanskrit after 1900 BC after the migrations to the UP-Bihar region and could have been transformed significantly by around $1700 B C=>$ were not written languages because the Brahminical orthodoxy prevented them from emerging as languages of power (However, there is evidence to establish that literacy always existed in the Gangetic plains in pockets) => they were re-popularized after the emergence of Buddhism. => They were influenced by Greek, Persian and other languages 
subsequently and by Sanskrit, many times.

Also,

The Prakrits of the Gangetic plains would have been de-synthesized from the Prakrits of the Indus after $1900 B C=>$ There would have been a re-synthesis after Aryan culture spread back into Pakistan after $1600 \mathrm{BC}$.

Prakrits (which were already influenced by Sanskrit) influenced South Indian languages as a result of contacts between the North and the South

Similarly,

Sanskrit particularly Classical Sanskrit, which itself was born as a liturgical language influenced Dravidian languages

Authors note: Skeptics are welcome to read our paper on literacy in post-Harappan India to appreciate how dates of the origin of an ancestor of Brahmi in India tally perfectly with this model, down to the very last century.

\subsection{Proposed Methods to Construct the Spoken Languages of the Harappans}

Proposed methods to construct the spoken languages of the Harappans are given below. This section would serve to illustrate the kind of innovative solutions that are possible.

We proposed the following methods for the reconstruction of the languages of the Indus:

Vedic Sanskrit - PIE influences - West Asian influences (Recommended!)

This is because, Vedic Sanskrit would have had Central and West Asian influences, as explained, and the balance would therefore represent Indo-Semitic. This is because the North-west of India was the only significantly populated region at the time. This would be the simplest and most reliable method and would be far superior to the only existing approach. The arrived at can be easily broken up into language groups). The residual IE words may again be removed. If this throws up words pertaining to Munda or languages which did not exist in the region and period in question, these will again need to be removed.

Method B

Taking the base in NW Prakrit languages of Ancient times including Dardic languages after removing Sanskrit words in whatever form PLUS (Vedic Sanskrit - PIE - language influences from West Asia during the compilation of the RV)

This method can be used once the smoking guns presented as a part of this paper are employed and may assist in a much more comprehensive reconstruction.

Method C

Taking the Prakrits of North-Western India and removing PIE influences from them. This method can be used once the smoking guns presented as a part of this paper are employed

Method D

Taking modern IA languages of the region including Dardic languages and working backwards from the present is another approach we can recommend. If modern languages of the region are to be taken as the base, we must systematically remove all later influences starting from the present. The advantage of the second and third methods is probably that they would permit us to reconstruct the different languages spoken in the IVC separately. 


\section{Macrothink}

\section{Method E}

\section{Substratum of Sanskrit}

This is an already-existing controversial method and can throw wrong results. If this method is to be employed, these must be accompanied by checks and balances, and these are below. If proponents of this method argue for a particular language, say language A, they must provide proof of the following, and using independent methods. If they cannot provide proof, their conclusions may be termed speculative. If opponents are able to clearly prove that the following checks and balances disprove language A as the substratum, this hypothesis is deemed falsified because these words would have (a) belonged to the Gangetic plains as in the case of Munda (The RV was compiled there) or (b) would have been shared with South India much later in time as would have been the case with Dravidian:

(a) Proof of ancientness of language family - Proponents of the any hypothesis must prove using independent methods that the language (or the language family) in question existed in the region during the period of the Indus valley Civilization.

(b) They must also provide theories to explain the origin and the spread of these languages in these regions.

(c) They must also explain, wherever applicable, how these languages might have been annihilated, and this is all the more important because IE speakers would have been small.

(d) Must show how linguistic transformations from Harappan to Post-Harappan India had taken place, as we have in this paper, moreover, given the fact that immigrants would not have been large in number.

(e) Must show how cultural transformations from Harappan India to Post-Harappan India occurred in detail.

(f) Must be accompanied by as many additional checks and balances as possible, and we have identified five smoking guns in our present paper.

Method F

A combination of all the above.

\subsection{Checks and Balances While Reconstructing the Languages of the Harappans}

The five smoking guns: The following checks and balances must be adopted while reconstructing the languages of the Harappans. These will be self-explanatory to anyone who has read or previous papers and should serve to illustrate the kinds of innovative solutions that are possible

Smoking gun A

Vedic Sanskrit - PIE influences - West Asian influences $=$ North West Prakrits + Dardic languages + Proto-Burushaski (after removing later influences including Sanskrit influences, i.e working backwards)

If the above is approximately true, the remote ancestor of Prakrits hypothesis is true Otherwise, it is false. This is because a mass extinction of languages in the region could not have taken place given the small number of migrants.

Smoking gun B

The languages constructed from our methods need to be compared with later Prakrits to 


\section{Macrothink}

investigate whether Prakrits are independent languages or derivatives of Sanskrit.

If the result is positive the Remote Ancestor or Prakrits hypothesis is correct and the Dravidian Harappa hypothesis is wrong. This is again because a mass annihilation of languages could not have taken place in the region.

Smoking gun $\mathrm{C}$

Here is our third smoking gun: If the substratum of Sanskrit contains Dravidian words and these words are present in IA too, the Dravidian Harappa hypothesis is inconclusive. If the Dravidian Harappa hypothesis is wrong, we can explain borrowings as having arisen as a result of cultural contacts between North and South India at a much later point in time.

Smoking gun D

Vedic Sanskrit - PIE influences - West Asian influences $=$ Prakrits + Dardic languages + Proto-Burushaski (after removing later influences including Sanskrit influences, i.e working backwards)

If the above equation does not tally, the Dravidian Harappa hypothesis has a chance (but it must pass all the other tests we proposed). Otherwise, it may be incorrect.

If the Dravidian Harappa hypothesis is wrong, we can explain borrowings as having arisen due to cultural contacts between North and South India.

Smoking gun $\mathrm{E}$

Vedic Sanskrit - PIE influences - West Asian influences contains Dravidian words which do not exist in Indo-Aryan languages.

The Dravidian Harappa hypothesis has a chance (but it must pass all the other tests)

\subsection{Maps Showing the Transformation of Harappan India to Post-Harappan India}

Maps explaining how the transformation of Harappan India to Post-Harappan India took place are below. There was perhaps a small genetic input, and migrants had not yet identified themselves as Aryan. These maps are self-explanatory and the interplay between two streams of languages can be easily understood after one has read these maps. All the other concepts in our papers will become extremely clear too. 


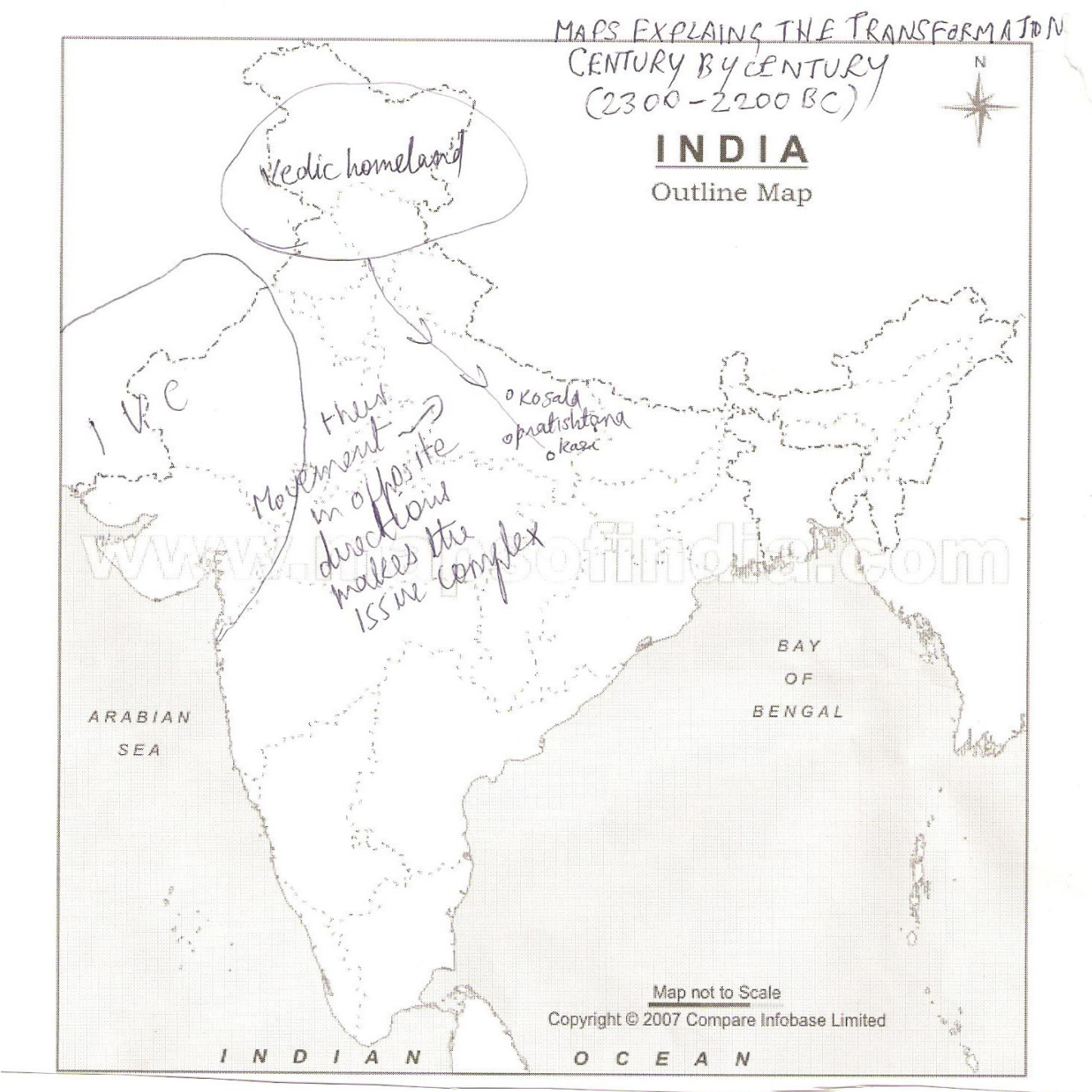

Note the eastward movement of the Indo-Aryans. They left the Himalayan region between $2200 \mathrm{BC}$ and $2300 \mathrm{BC}$ and moved along the Ganga and the Yamuna, through jungles. They founded many kingdoms there e.g (a) Pratishtana (Lunar Dynasty or Moon dynasty) (Later known as Prayag and then Allahabad (b) Kosala (Ayodhya) (c) Kasi (Benares or Varanasi).

Given that the number of migrants from Central Asia even in multiwave models would be limited to the populations there (it is unlikely that two people rode in per horse), and the fact that there were a small number of people in the Gangetic plains before $2200 \mathrm{BC}$ would suggest that the inhabitants of the IVC were ancestors of the people living in the UP-Bihar region. Even If we assume that the immigrants were small and were male (they would then have naturally lost their identity in the Himalayan region itself), we can convincingly explain the spread of IE culture century by century. 


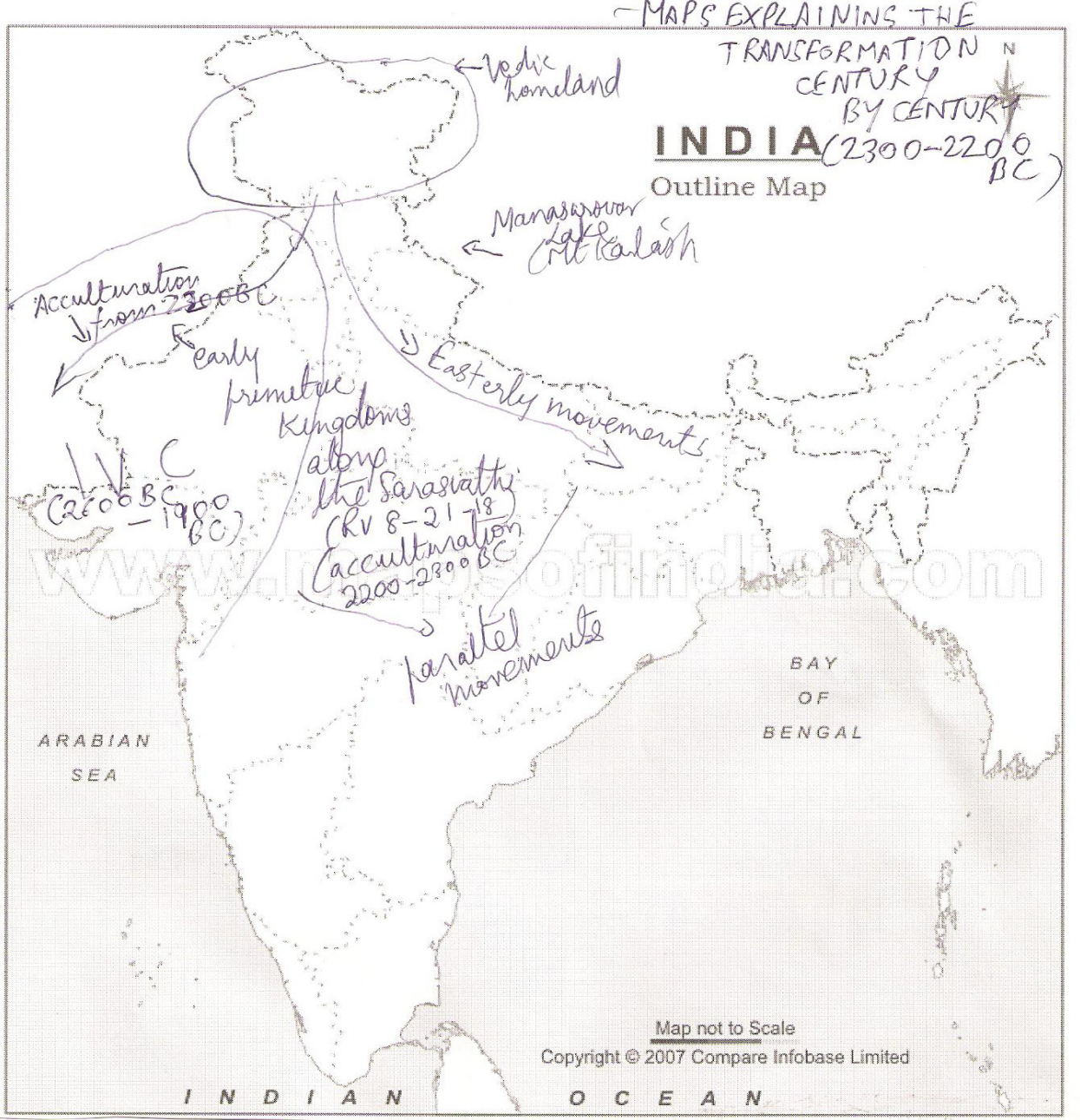

Note the parallel movements of the Indo-Aryans. Acculturation within the IVC would explain the Saraswat Brahmins (Note:It is necessary to understand the southward migrations of $1900 \mathrm{BC}$ well and the acculturation must have already begun before that), the presence of alien objects, the petty kingdoms along the Saraswathi river according to the RV and changes in Burial practices by $1900 \mathrm{Bc}$ - the Cemetery H culture may be as a result of a famine in $1900 \mathrm{BC}$ when the eastward movement started. It is fascinating to understand, always using logic and reasoning, taking century by century snapshots, how composite Indian culture got formed starting from the Kot Diji phase of $3300 \mathrm{BC}$. This would be a fascinating voyage of understanding. 
MAPS EXPZAINING THE

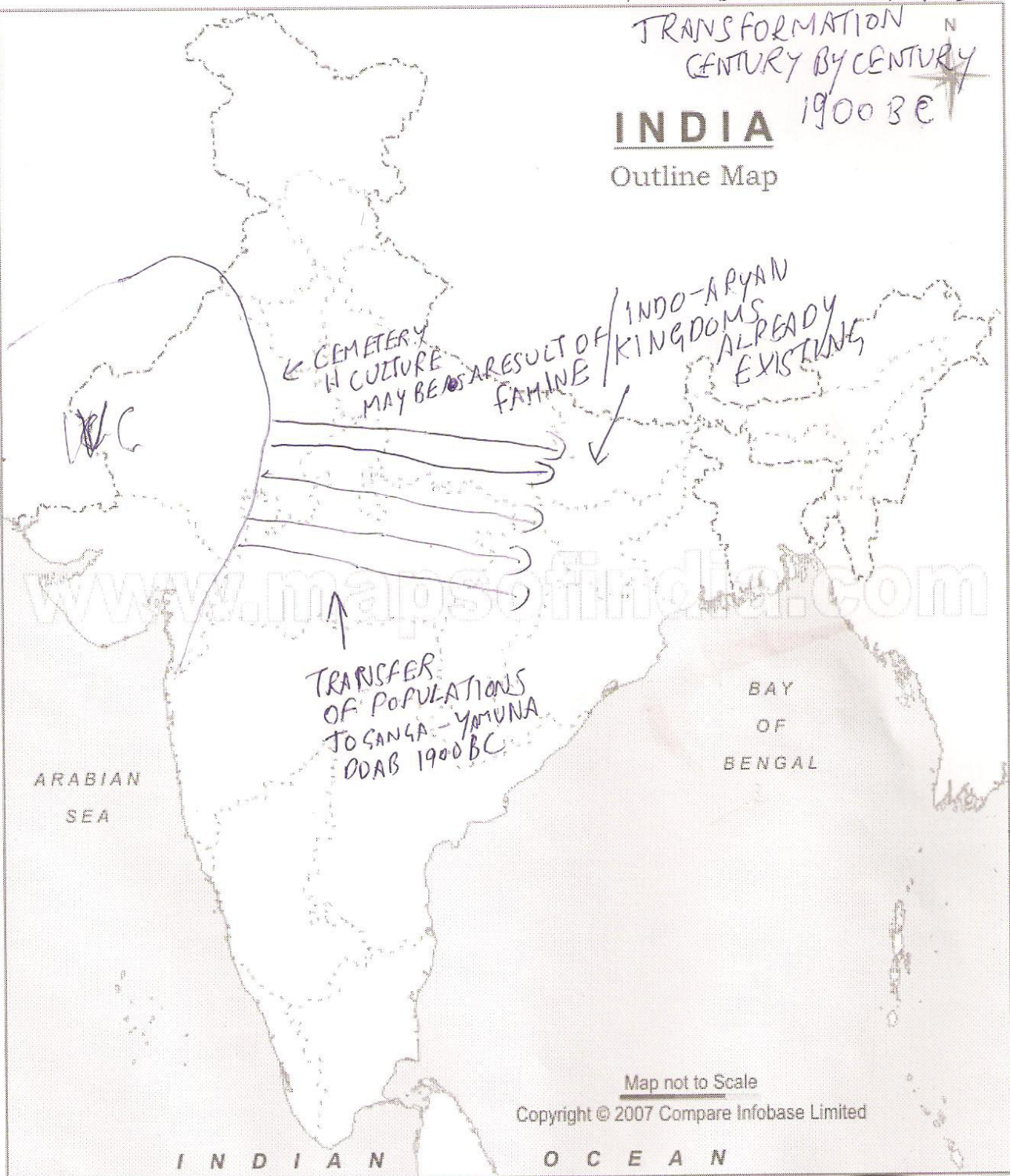

The Saraswathi river must have dried up around $1900 \mathrm{BC}$ as suggested by the ASI because this The Saraswathiver must have dred up around 1000 BC as suggested by the ASI because this date talles with the Cemetery $H$ culture and the beginning of the decline of the $\mathrm{NC}$ well. The this river. 


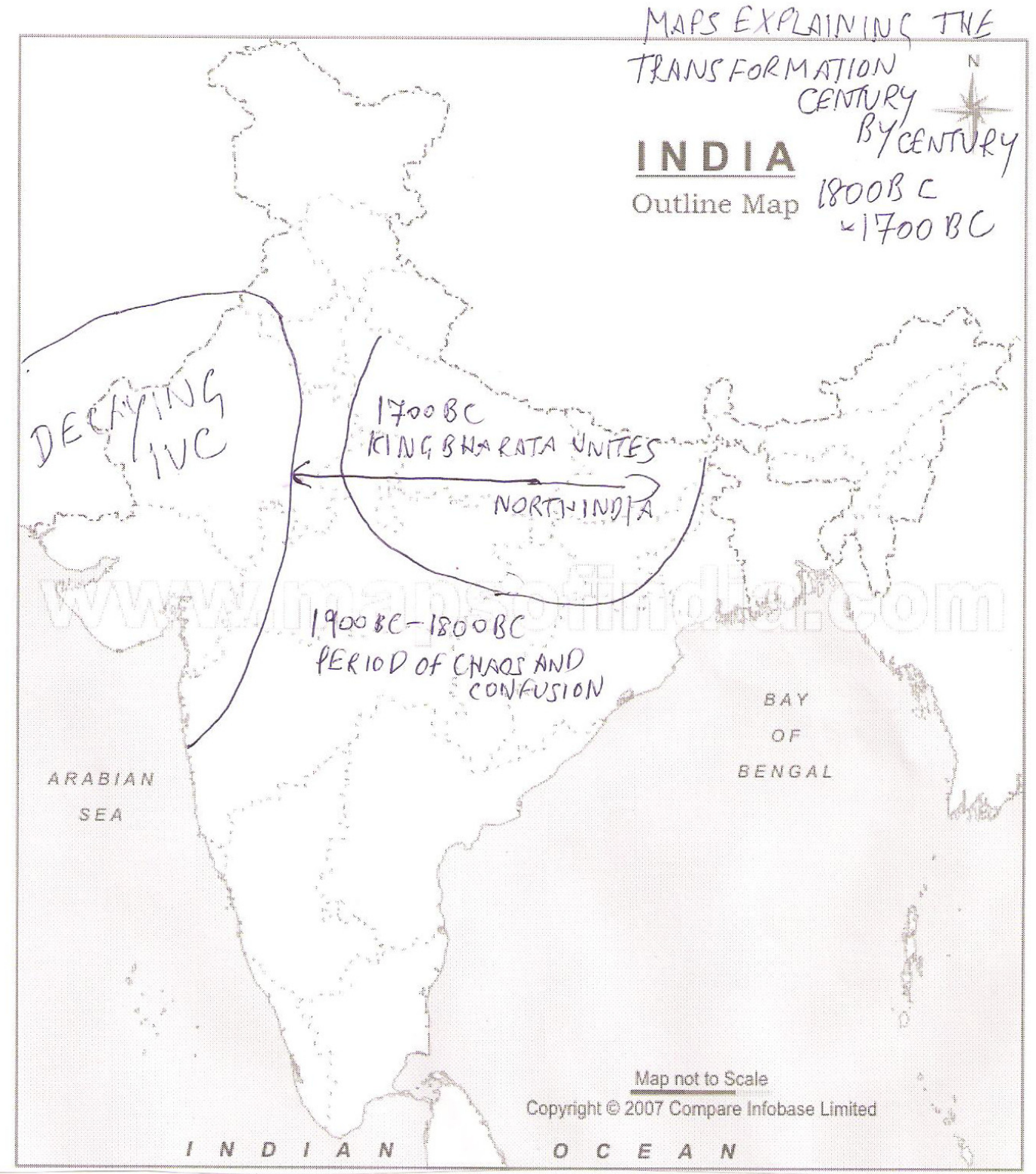

There is a wealth of information about the reign of King Bharata as known through traditional sources. It is believed that his reign marked a major turning point in the expansion of Indo-Aryan power. 


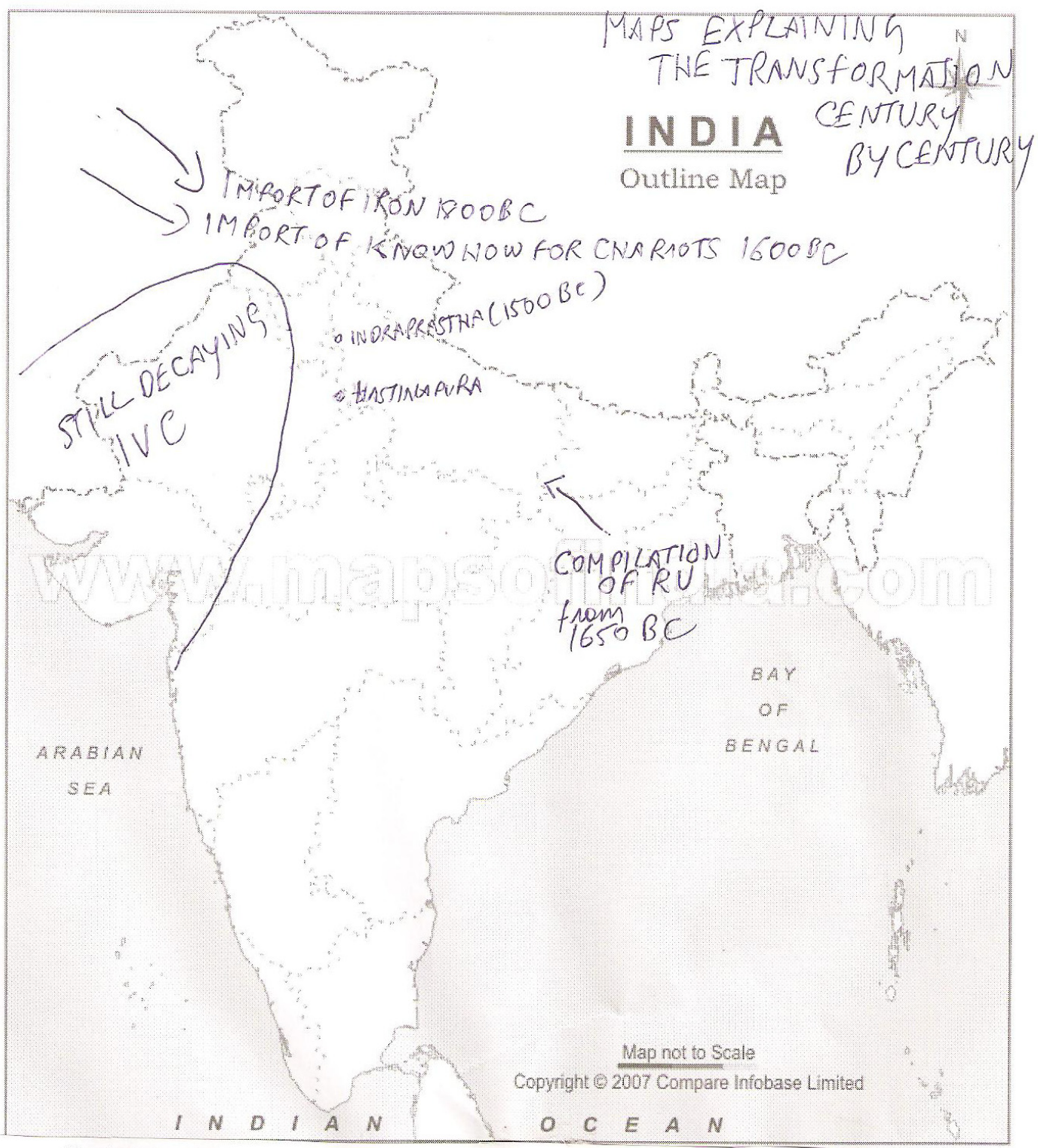

Trade and cultural contacts with West Asia began around $1800 \mathrm{BC}$, or perhaps a little later than
this. Iron was perhaps Indraprastha and Hastinapura were found $1800 \mathrm{BC}$ and knowhow for chariots around $1600 \mathrm{BC}$. means that several events were taking place in paral $1500 \mathrm{BC}$. The concurrent dating paradigm about the Gangetic plains and fit it into this model. 


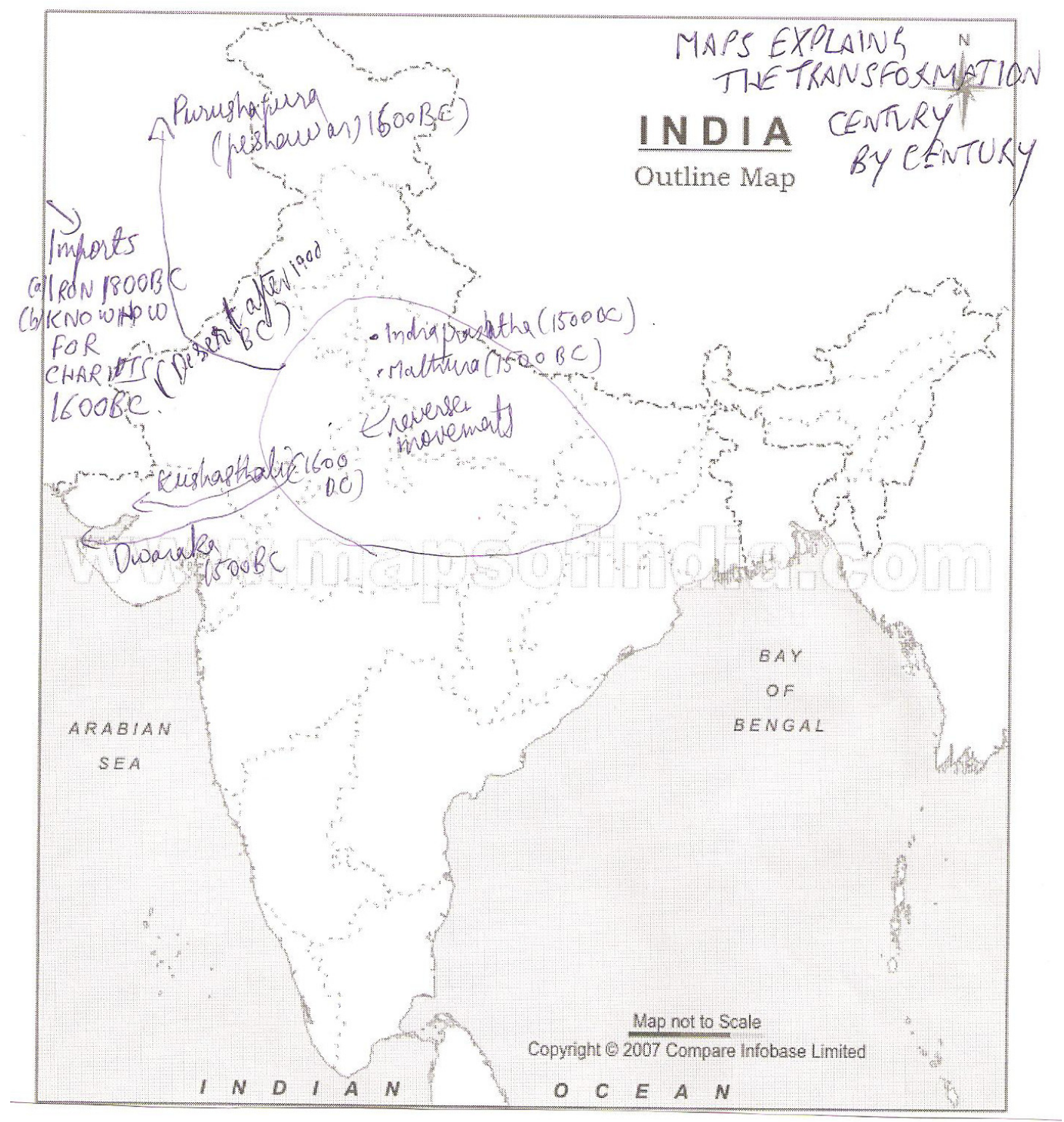

Note how the Indo-Aryan kingdoms moved back close to the decaying IVC. However, the region between the two became a desert after the eastward migrations of $1900 \mathrm{BC}$, when the populations of the IVC were split up between the Gangetic plains and the Indus.

However, this model differs fundamentally from earlier models in many respects: While IE culture spread, they killed their own language and made it (i) a liturgical language and (ii) a lingua franca of the elite. Secondly, Prakrits did not derive from Sanskrit but closely interacted with them over millenia. This theory if accepted, would change the way we look at IA languages and undestand the probable distant relationships between modern IA languages like Punjabi, Gujarati and the languages of the Indus. Note our century by century depiction of the transformation. 


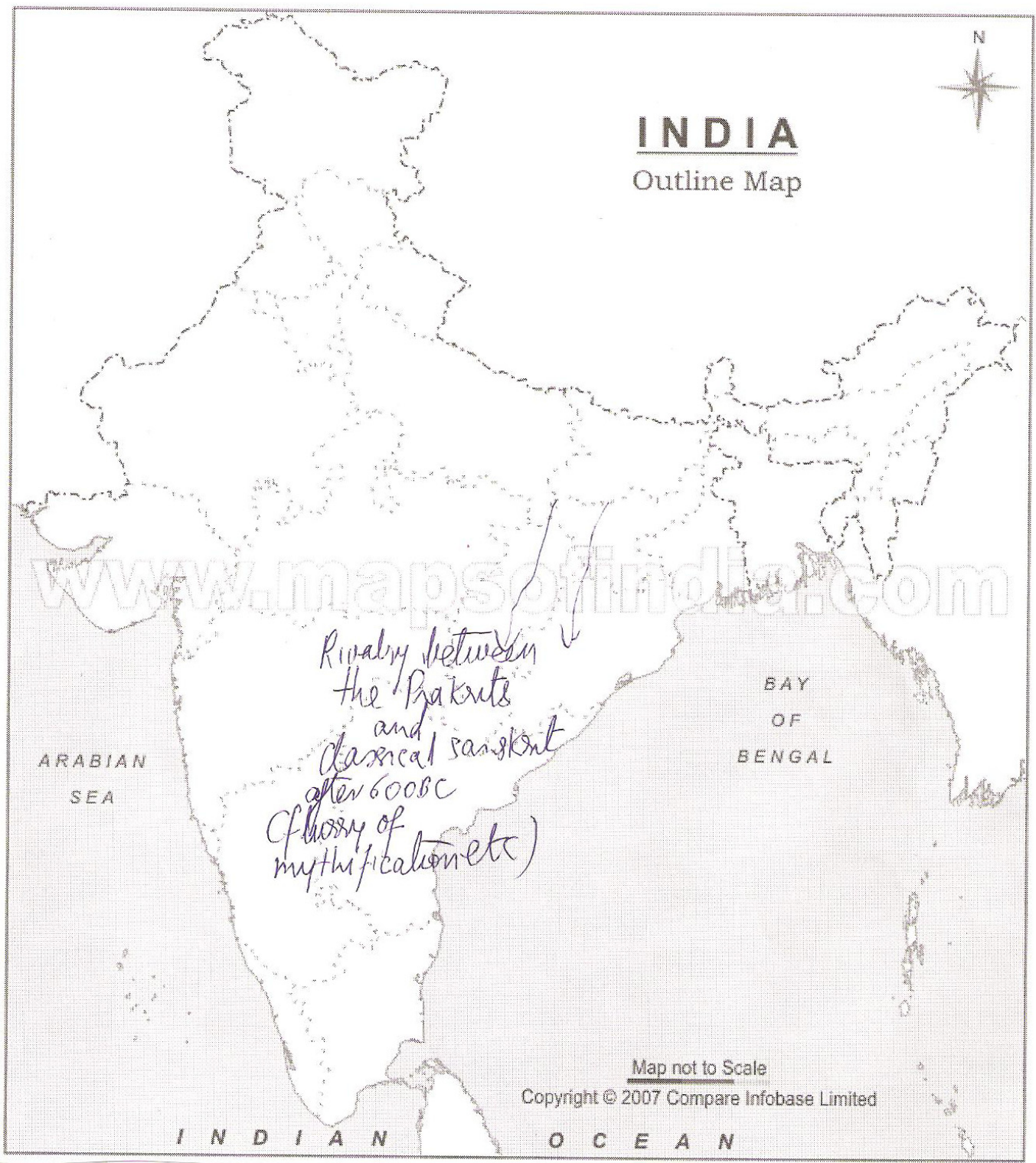

Note the changes to Indian culture as a result of the competition between Buddhism and the threatened Vedic orthodoxy. Classical Sanskrit began to emerge after $500 \mathrm{BC}$. Another trigger was perhaps Asoka's conversion to Buddhism around $200 \mathrm{BC}$ which resulted in composition of new works and significant changes to religion. 


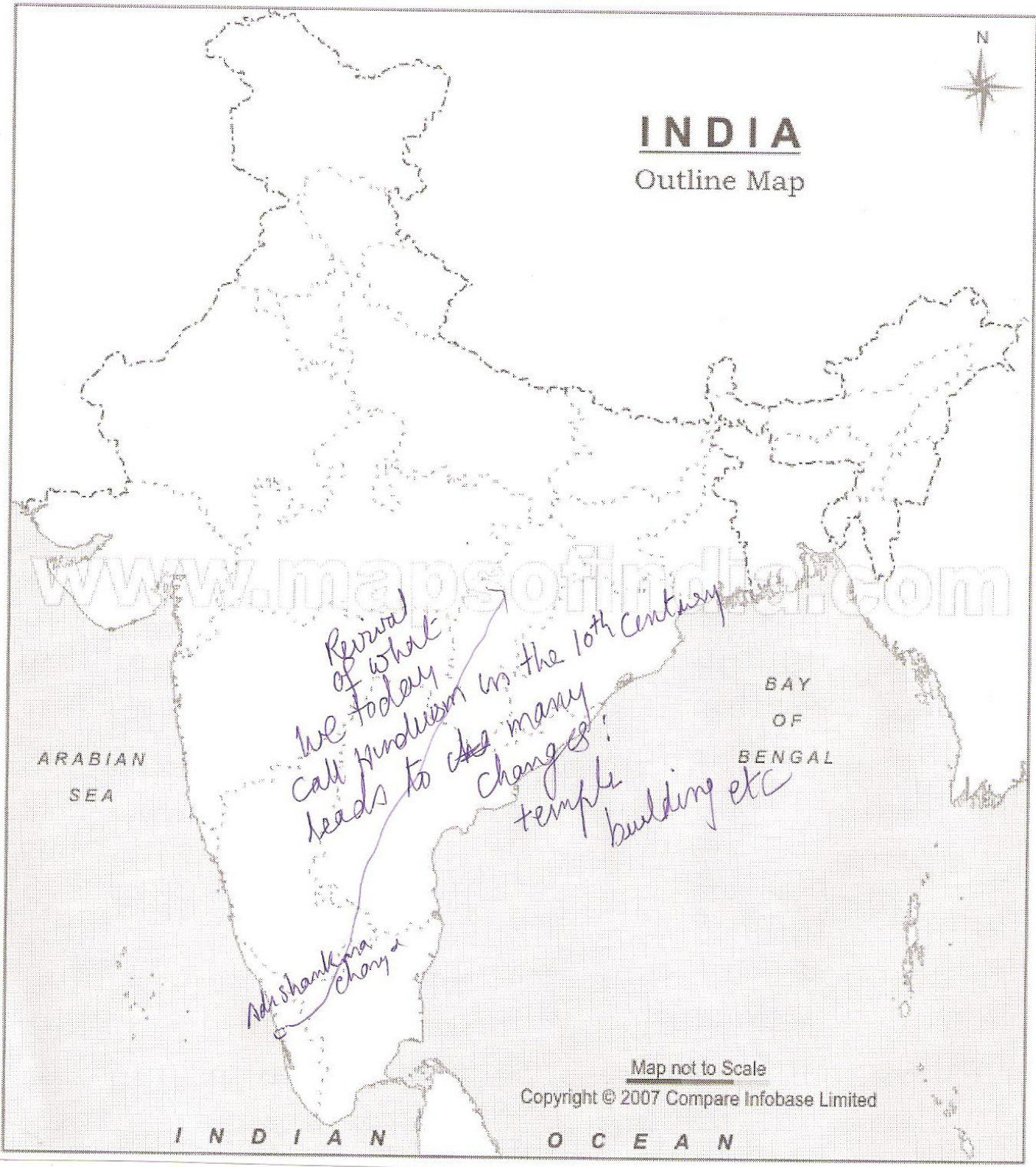

Around $800 \mathrm{AD}$, the religion that is now called Hinduism appears to have been threatened by Buddhism again. Adi Sankaracarya was an Indian philosopher who

popularized the Vedas. The period appears to have been marked by many changes to religion such as temple building. 


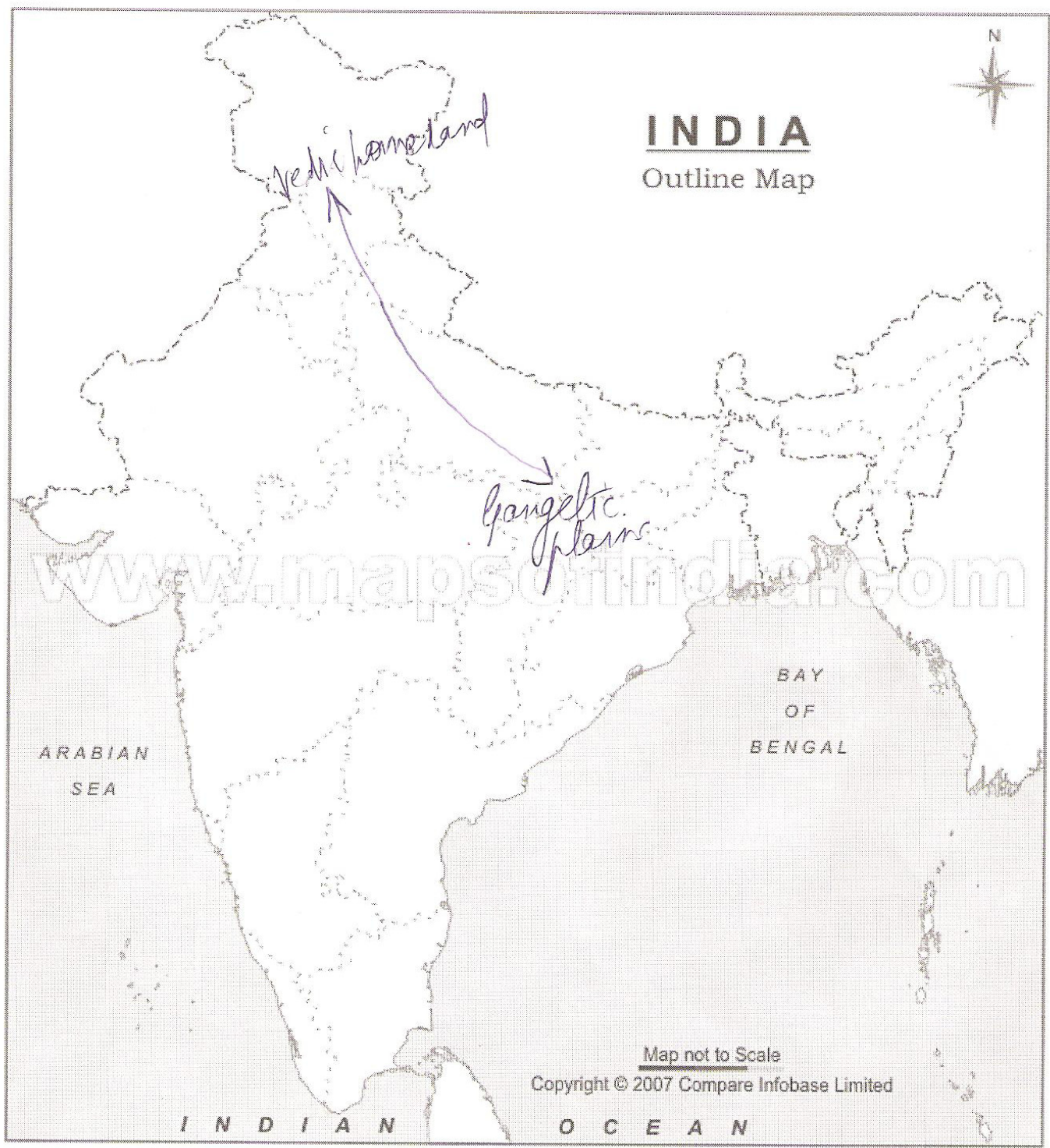

Contacts between the Gangetic plains and the Vedic homeland will explain the retention of ames of plants found in cool climates when the Vedas were compiled. 


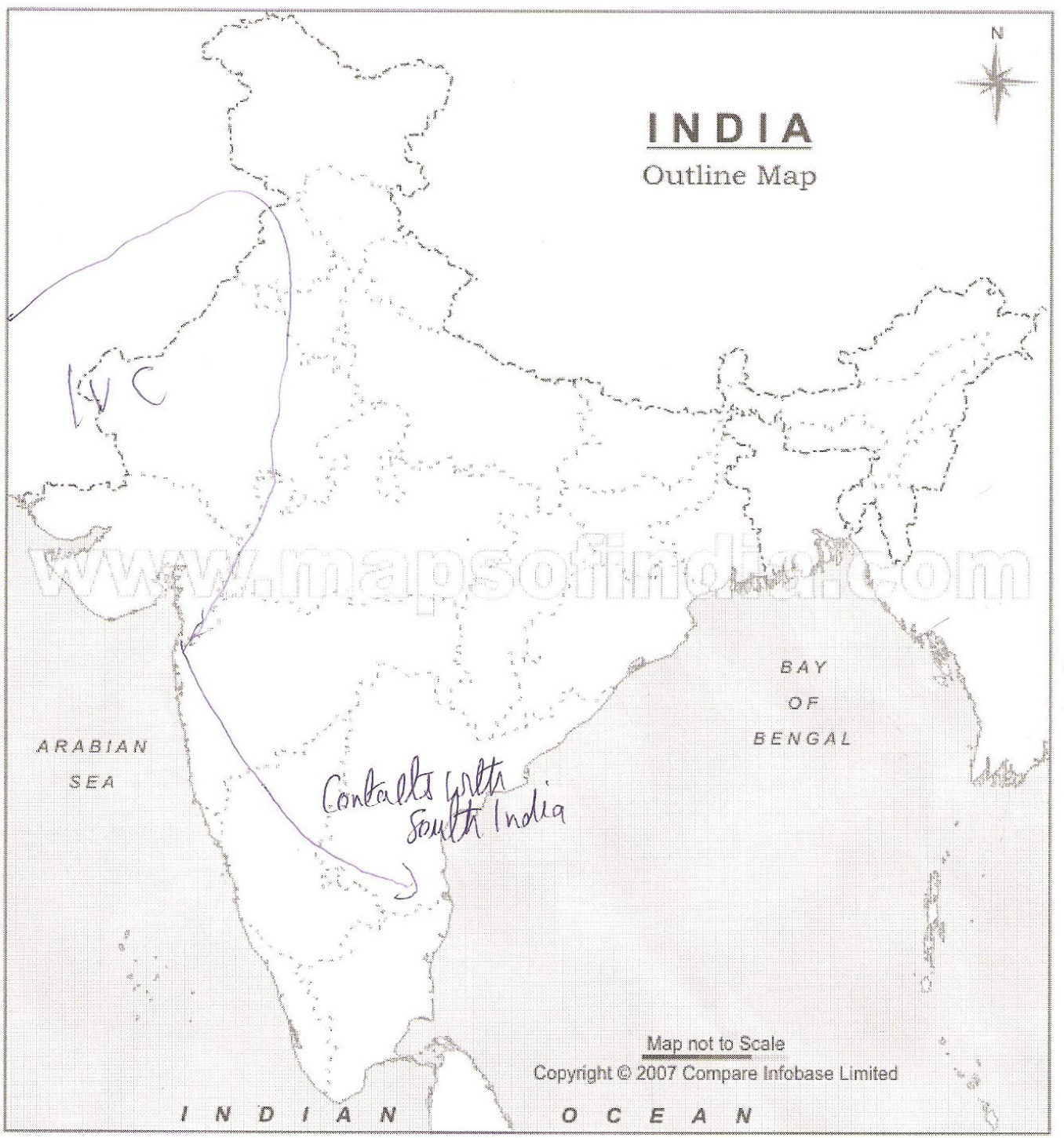

Direct contacts between the IVC and the South of India will explain many aspects of Indian culture not accounted for by our Nine phases as discussed in this paper. The problem with the Dravidian Harappa hypothesis is that all aspects of Dravidian culture are not represented in the IVC.

Very important explanatory notes to maps on previous pages:

(1) Note how the desynthesis between the Harappan culture and the territory further east which led to the formation of an independent culture to the East of the Saraswathi took place. 


\section{Macrothink}

This happened due to the desertification of the present-day Rajasthan region after 1900 BC. Understanding this would be crucial to understanding this model.

(2) Conflicts or violent incidents would only have been internal as discussed in the paper 'Syncretism and Acculturation in Ancient India'. (Parts one and two).

(3) Note: It is not necessary to justify migrations in the second millennium on account of the mention of plants found in cooler climates in the RV, and these is enough evidence to adduce that migrations did not happen in the Second Millennium BC.

\subsection{Additional Maps}

Page 1 of 1

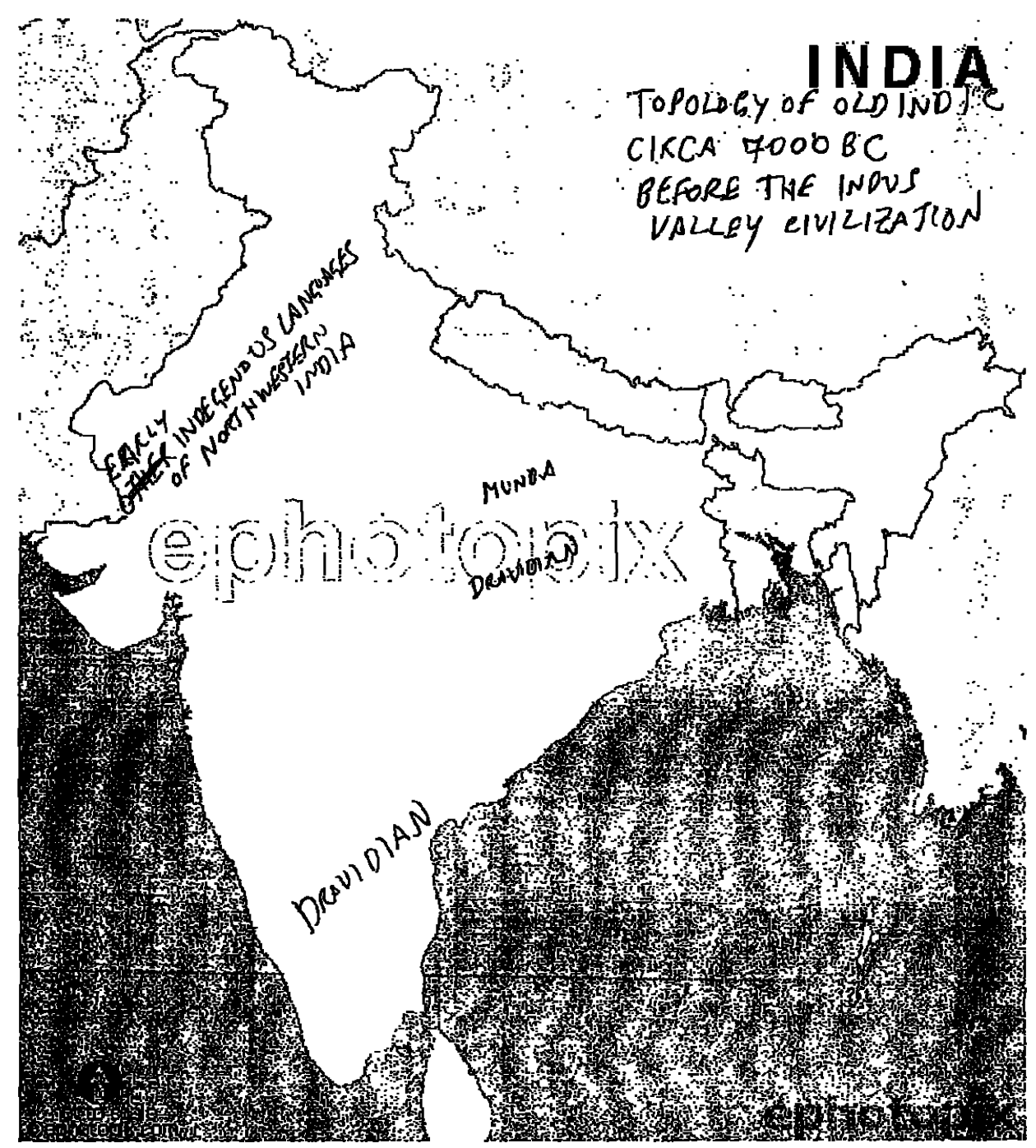

Explanatory notes to map on previous page (Topology of Old Indic before the IVC):

The Indus Valley was only gradually settled in from Baluchistan, and the progenitor cultures of the IVC were in Mehrgarh and the surrounding regions. These languages would have therefore been related to the ancestors of the languages further West such as Semitic, Old Elamite and the contemporaneous languages of Iran. Other indigenous languages would have 
perhaps existed in the Indus Valley before the Mature Harappan phase. These can be identified perhaps, if a 'layer by layer' approach is adopted. The languages of the IVC, when reconstructed from the methods proposed in our paper, can be compared with the languages further west, to identify the differences between the two. Dravidian languages would have existed in the South of India since time immemorial, and in parts of Central India. Munda languages would have been spoken further north, in the Gangetic plains and in Central India.

Page 1 of 1

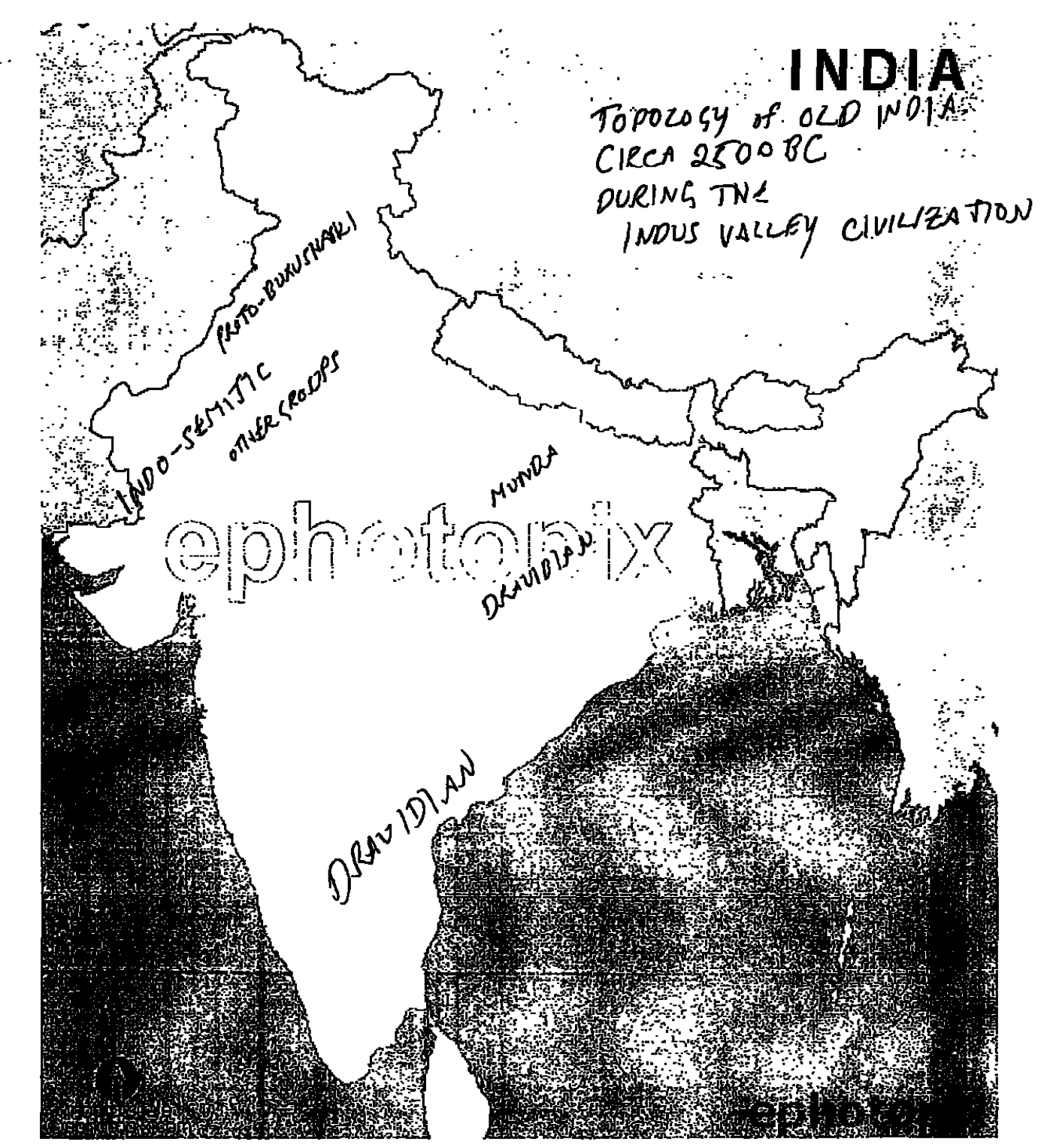

Explanatory notes to map on previous page (Topology of Old Indic during the Mature Harappan phase):

By the time of the mature Harappan phase, the languages of Baluchistan would have synthesized with the various languages already spoken in the Indus valley to give rise to the languages spoken in the Indus valley civilization. There would have been undoubtedly many 


\section{MInstitute ${ }_{\text {Mink }}^{\text {Macrothink }}$}

languages belonging to many language families spoken here, such as proto-Burusharski, and these can be reconstructed from the methods proposed in our paper. We have collectively referred to these as Indo-Semitic

Page 1 of 1

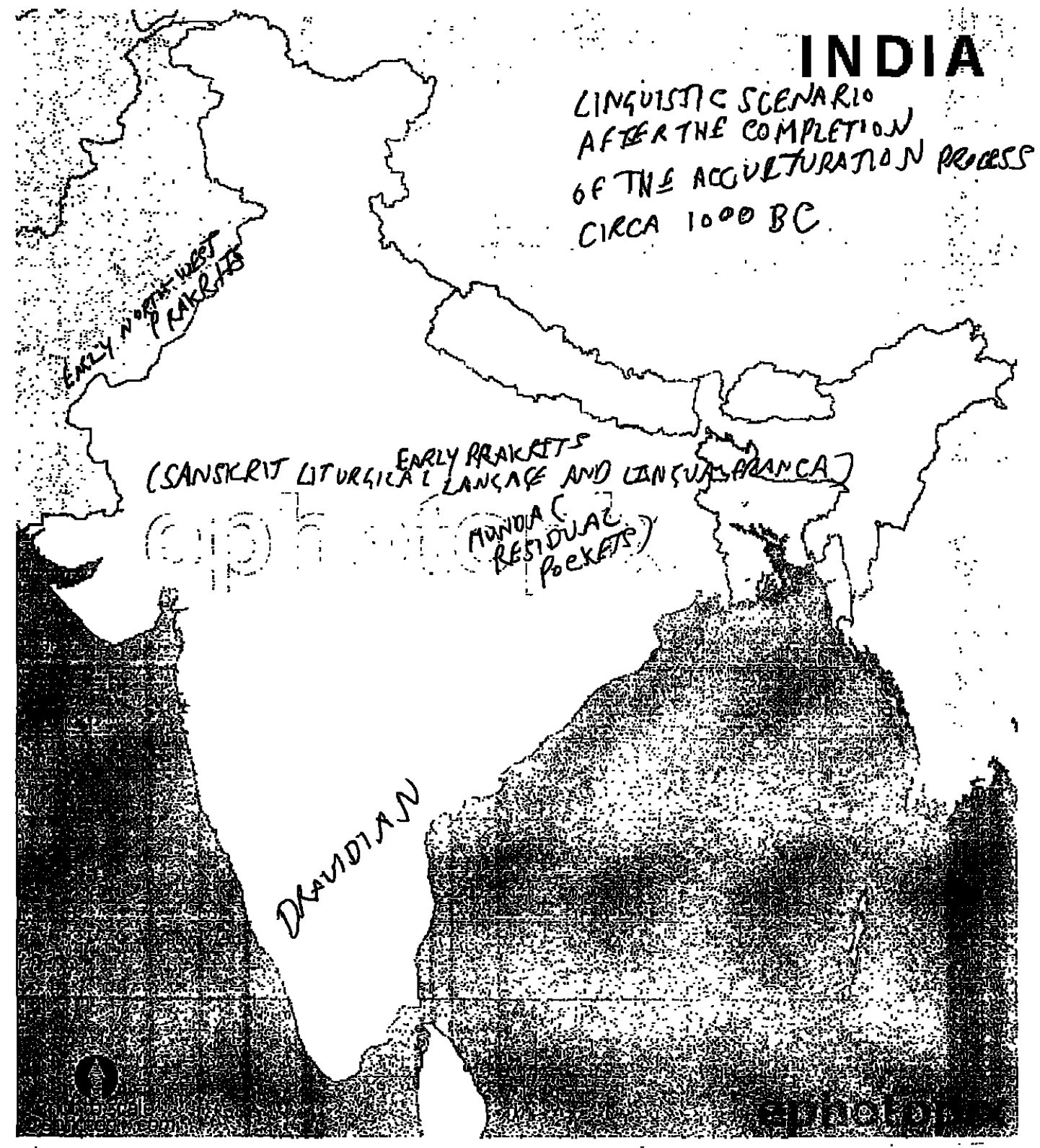

Explanatory notes to map on previous page:

By $1000 \mathrm{BC}$, the process of acculturation was complete and the cities of the IVC were buried and long-forgotten. The status of Dravidian languages in the South of India remained relatively unchanged. In the centuries to come, these would be impacted by cultural inputs from the North. Munda languages would be consigned to residual pockets in North and Central India, and may have vanished from the river valleys, where they may also have been 


\section{Macrothink}

spoken before migrations from the Indus took place in $1900 \mathrm{BC}$. The languages of the IVC evolved into the early Prakrits of the Gangetic plains and the early NW Prakrits of NW India. Vedic Sanskrit by this time, was only a liturgical language, a lingua franca of the elite, and was slowly becoming a cultural symbol of Ancient India.

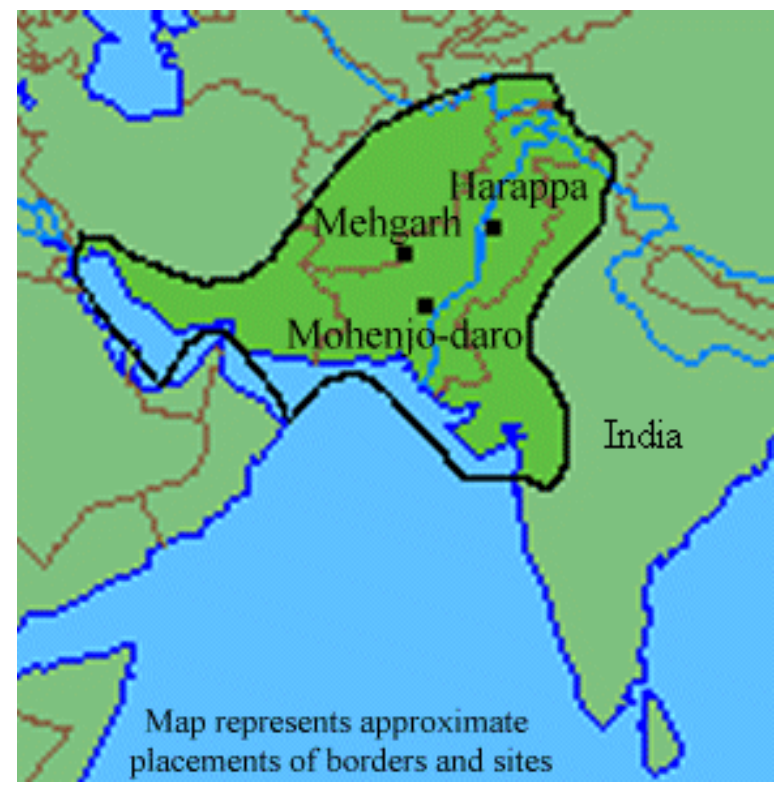

Note that most regions of Baluchistan speak languages that are not labelled Indic. Pashto is itself a part of the Eastern Iranian family of languages. (and is quite distinct from Indic). As the IVC was only gradually settled in from the Baluchi hills, the languages of the IVC (core region of the IVC) were already Indic. This is because there was a time gap on 4400 years between the start of Mehrgarh phase one and the mature Harappan. These were obviously the present-day ancestors of languages of the region! Map: http://indianhistory.info/indus.htm

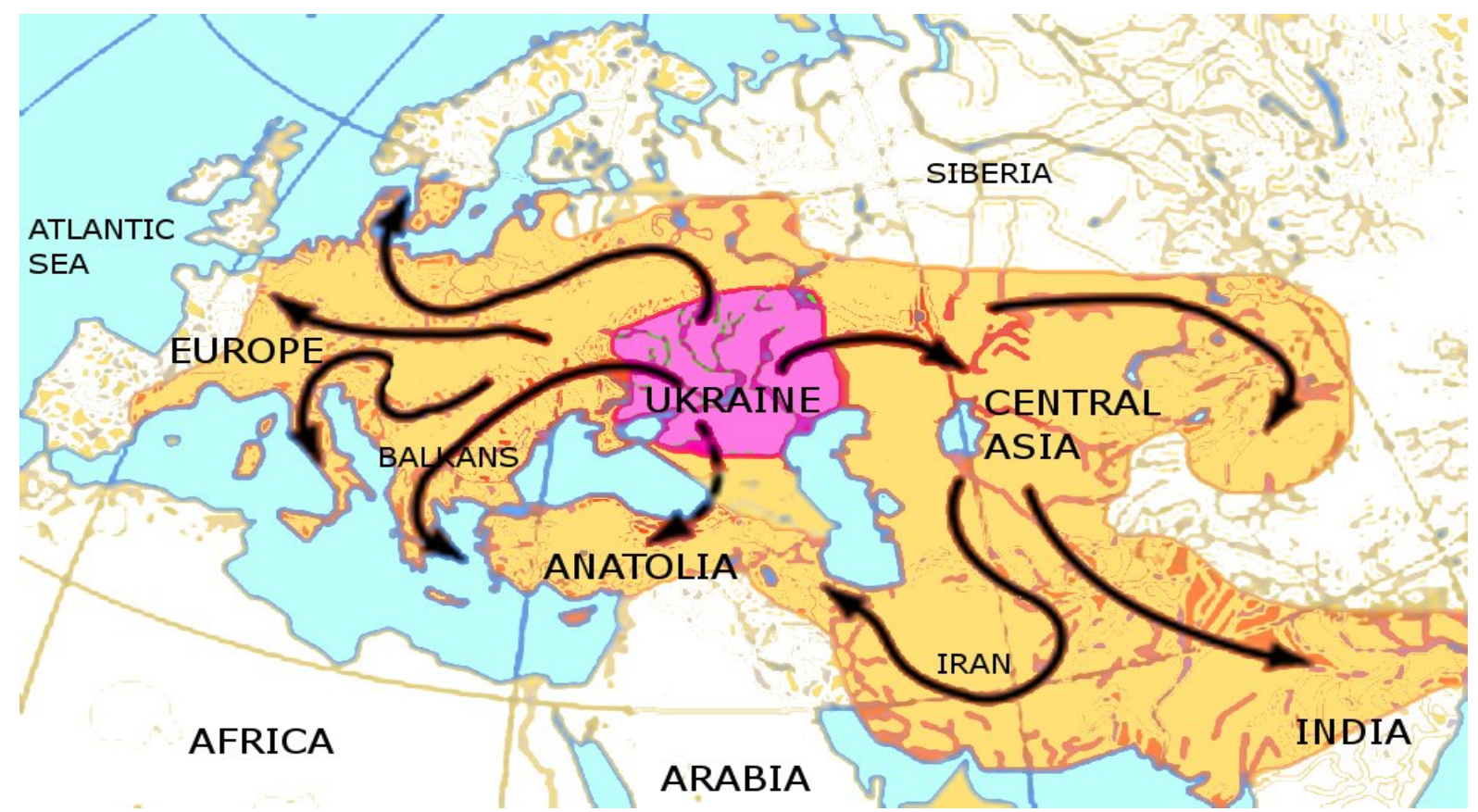

Note the spread of IE peoples according to the Kurgan hypothesis. The location of the Urheimat is irrelevant to our paper. There may have been more than one; the domestication of 


\section{Macrothink}

Journal of Social Science Studies

ISSN 2329-9150

2016, Vol. 3, No. 1

the horse may have been a trigger for movements. The number of IE speakers into India would have been small in comparison with the large population of the IVC. (Map: www.protogermanic.com)

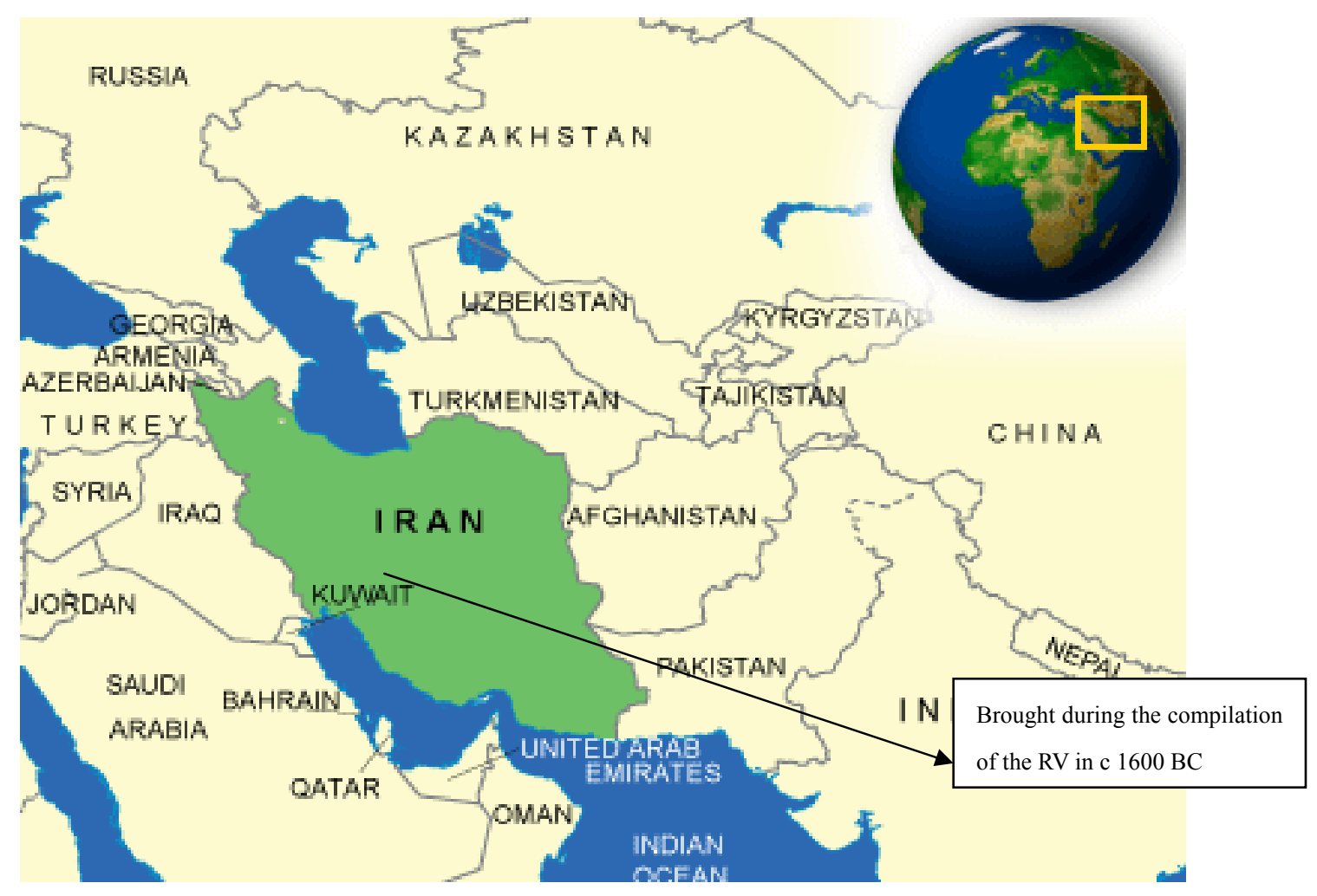

Note that the term Aryan would have been brought from Iran to India during the compilation of the RV between $1700 \mathrm{BC}$ and $1500 \mathrm{BC}$. In the RV, the term Aryan was cultural. The small numbers of people who had migrated from an Urheimat much earlier in $2750 \mathrm{BC}$ would not have called themselves Aryan. (Map: www.countryreports.org) 


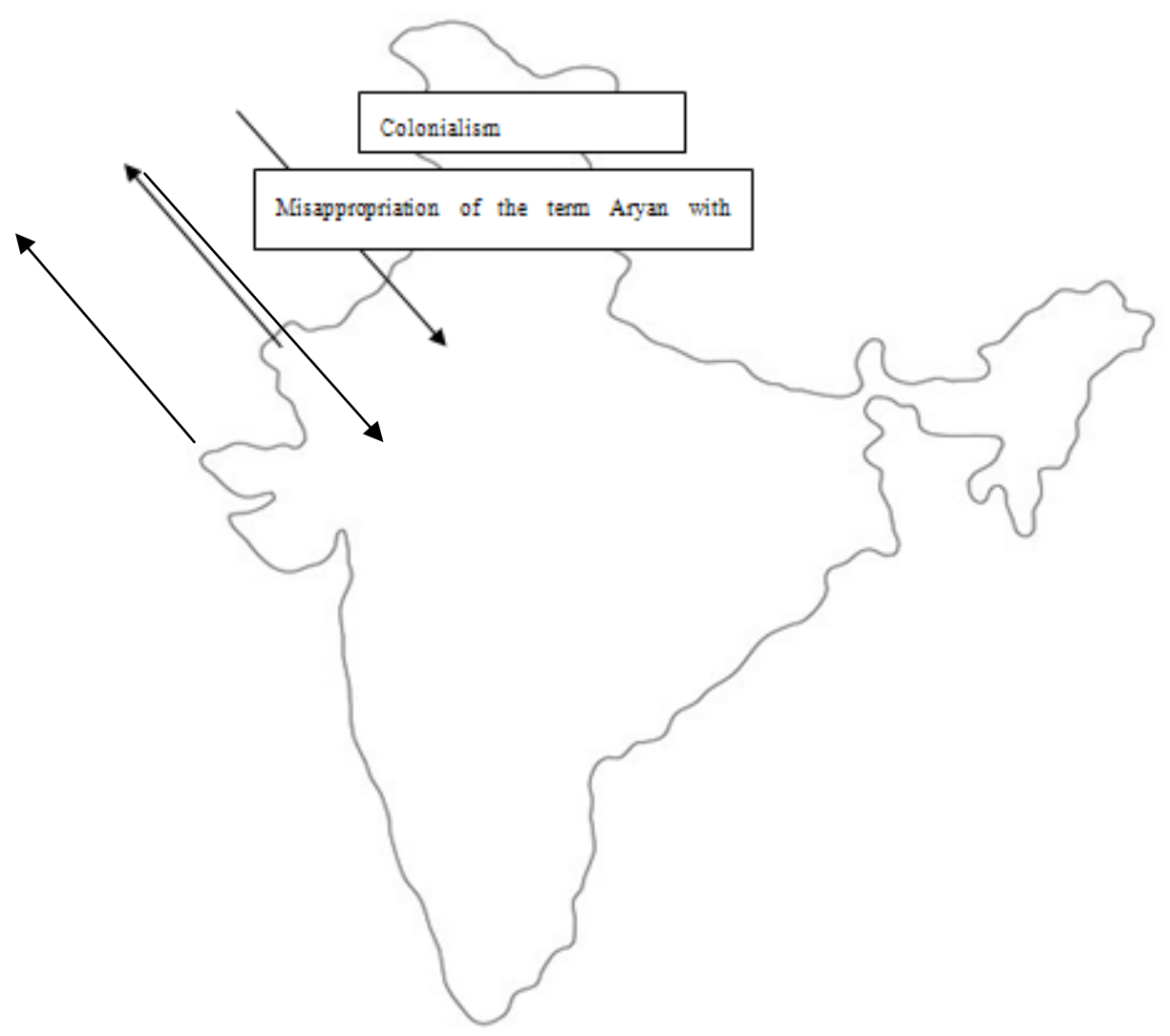

Note that the misappropriation of the term 'Aryan' to mean a race began only in the nineteenth century. Its usage in the West was virtually unknown before the eighteenth century. Exceptions were its usage in specific contexts. An example being Megasthenes' use of the term Arianois to refer to countries close to India.

\section{References}

On the internal classification of Indo-European languages: survey by Václav Blažek

Syncretism and Acculturation in Ancient India: A new Nine phase acculturation model explaining the process of transfer of power from the Harappans to the Indo Aryans Part One Sujay Rao Mandavilli ICFAI Journal of history and culture January 2009

Syncretism and Acculturation in Ancient India: A new Nine phase acculturation model explaining the process of transfer of power from the Harappans to the Indo Aryans Part Two Sujay Rao Mandavilli ICFAI Journal of history and culture January 2010

Twenty-first century clouds over Indo-European homelands: J P Mallory Queens University, Belfast

The Indo-European Family - The linguistic evidence by Brian D. Joseph, The Ohio State University

History of Civilizations of Central Asia, Volume I and II (UNESCO Publishing)

Cambridge Textbooks in Linguistics: Sociolinguistics Second Edition R.A Hudson 
Cambridge University Press 2001

General Linguistics and Indo-European Reconstruction Frederik Kortlandt

Observations on language spread in multi-lingual societies: Lessons learnt from a study of Ancient and Modern India Sujay Rao Mandavilli, Elk Asia Pacific Journal of Social studies, June 2015

Alexandre Fran, cois. Trees, Waves and Linkages: Models of Language Diversification. Bowern, Claire; Evans, Bethwyn. The Routledge Handbook of Historical Linguistics, Routledge, pp.161189, 2014, 978-0-41552-789-7.

Classification and Evolution in Biology, Linguistics and the History of Science, Heiner Fangerau, Hnas Geisler, Thorsten Halling and William Martin, Franz Steiner Verlag, 2013

Trees, Waves and Linkages: Models of Language Diversification Alexandre Francois

The Routledge Handbook of Historical Linguistics, Edited by Claire Bowern and Bethwyn Evans, Routledge

Indo-European linguistics: An introduction James Clarkson Cambridge

Indo-Europeanization: The Seven dimensions in the study of a never-ending process Harald Haarmann, Institute of Archaeomythology, Sebastopol USA

The reconfirmation and reinforcement of the Indus script thesis: A logical assessment and inquiry as to the elusive and enigmatic nature of this script Sujay Rao Mandavilli Published in the ICFAI Journal of History and Culture, January 2011

Alphabetic scripts and other forms of literacy in PostHarappan India: A logical assessment and inquiry as to the origin and extent of literacy in Post-Harappan India Sujay Rao Mandavilli International Journal of Philosophy and Social Sciences, October 2012

Why the Indus Script WAS true writing and why a larger corpus of texts existed in the Indus Valley civilization: Simple proof addressed to mainstream researchers \& archaeologists Sujay Rao Mandavilli International Journal of Philosophy and Social Sciences, March 2013

Joseph Arthur compte de Gobineau, Essai sur l'inegalite des races humaine, 4 vols (Paris:Firmin Didot Freres

Max Mueller, Biographies of Words and the Home of the Aryas, 1888, pg 120

"The demise of the Dravidian, Vedic and Paramunda Indus hypotheses: A brief explanation as to why these three hypotheses are no longer tenable" Sujay Rao Mandavilli (A supplement to the authors papers published in the ICFAI University press on the Aryan problem)

The home of the Aryans, Michael Witzel Harvard University

Autochthonous Aryans? The Evidence from Old Indian and Iranian Texts. Michael Witzel Harvard University

Savarkar V.D, Who is a Hindu? Page 68, 1969

Understanding language change April M.S MacMahon, Cambridge University Press 


\section{Macrothink}

Journal of Social Science Studies

ISSN 2329-9150

\section{Copyright Disclaimer}

Copyright reserved by the author(s).

This article is an open-access article distributed under the terms and conditions of the Creative Commons Attribution license (http://creativecommons.org/licenses/by/3.0/). 